\title{
Particle trajectories beneath wave-current interaction in a two-dimensional field
}

\author{
Y.-Y. Chen ${ }^{1,3}$, H.-C. Hsu ${ }^{2}$, and H.-H. Hwung ${ }^{3}$ \\ ${ }^{1}$ Dept. of Marine Environment and Eng., National Sun Yat-Sen Univ., Kaohsiung 804, Taiwan \\ ${ }^{2}$ Tainan Hydraulics Laboratory, National Cheng Kung Univ., Tainan 701, Taiwan \\ ${ }^{3}$ Dept. of Hydraulic and Ocean Eng., National Cheng Kung Univ., Tainan 701, Taiwan
}

Correspondence to: H.-C. Hsu (hchsu@ thl.ncku.edu.tw)

Received: 25 October 2011 - Revised: 29 January 2012 - Accepted: 6 February 2012 - Published: 16 March 2012

\begin{abstract}
Within the Lagrangian reference framework we present a third-order trajectory solution for water particles in a two-dimensional wave-current interaction flow. The explicit parametric solution highlights the trajectory of a water particle and the wave kinematics above the mean water level and within a vertical water column, which were calculated previously by an approximation method using an Eulerian approach. Mass transport associated with a particle displacement can now be obtained directly in Lagrangian form without using the transformation from Eulerian to Lagrangian coordinates. In particular, the Lagrangian wave frequency and the Lagrangian mean level of particle motion can also be obtained, which are different from those in an Eulerian description. A series of laboratory experiments are performed to measure the trajectories of particles. By comparing the present asymptotic solution with laboratory experiments data, it is found that theoretical results show excellent agreement with experimental data. Moreover, the influence of a following current is found to increase the relative horizontal distance traveled by a water particle, while the converse is true in the case of an opposing current.
\end{abstract}

\section{Introduction}

The problem of nonlinear water waves propagating through areas containing tidal, ocean or discharge current is an important issue in marine environments. The interaction between these flows plays vital roles in many aspect of coastal and ocean engineering, for example forces due to such flow fields on fixed or floating offshore wind turbines, sediment transport, contaminant and nutrient dispersion. The phenomenon of wave-current interaction has been studied extensively since the 1970s. Several theoretical solutions for waves on currents with uniform or sheared profiles have been well documented in the review articles of Peregrine (1976), Jonsson (1990) and Thomas and Klopman (1997). Reports are also available on experimental studies for combined wave and current covering various aspects of this problem (Brevik, 1980; Constantin and Strauss, 2004; Kemp and Simons, 1982, 1988; Thomas, 1981, 1990).

Most previous theories dealing with wave-current interactions have employed the Eulerian description, in which the free surface fluctuations can be expressed in a Taylor series expansion relative to a fixed water level (i.e., the still water level). This implicitly assumes that the surface profile of a wave is a differentiable single-valued function. Unlike the Eulerian free surface, which is given as an implicit function, a Lagrangian surface is described through a parametric representation of the position of a particle. The use of Lagrangian coordinates yields the only known nontrivial exact solutions to the governing equations for gravity water waves (i.e., Gerstner's solution for deep-water waves (Gerstner, 1802) and a recently found edge wave solution along a sloping beach (Constantin, 2001) which was extended to stratified flows (R. Stuhlmeier, 2012)). The main advantage of such a description is to allow better flexibility for describing the actual shape of the ocean surface, which will be demonstrated later in this paper. Based on this reason, it has been shown that the Lagrangian description is more appropriate for the motion of the limiting free surface, which cannot be captured by the classical Eulerian solutions (Biesel, 1952; Chen et al., 2006; Naciri and Mei, 1992). However, reports on this notable improvement using Lagrangian description are rather limited.

The first water wave theory in Lagrangian coordinates in which the flow possesses finite vorticity was presented by Gerstner (1802), which was re-discovered by Rankine (1863) and the modern discussion by Constantin (2001) and Henry (2008). Miche (1944) proposed perturbation Lagrangian solutions to the second order for a gravity wave. Pierson (1962) applied the Navier-Stokes equation to the 
deep water waves in the Lagrangian formulae and obtained the first-order Lagrangian solution. Sanderson (1985) obtained second-order solutions for small amplitude internal waves in a Lagrangian coordinate system. $\mathrm{Ng}$ (2004) re-examined the problem of mass transport due to partial standing waves in one and two layer fluids. Buldakov et al. (2006) developed a Lagrangian asymptotic formulation up to the fifth order for nonlinear water waves in deep water. Clamond (2007) obtained a third-order Lagrangian solution for gravity waves in finite-depth water and a seventh-order solution for deep water waves. To date, only a limited few theoretical solutions are derived for wave-current interaction in Lagrangian coordinates. Umeyama (2010) gave a wrong third-order solution of particle trajectory, which did not include Lagrangian wave frequency and Lagrangian mean level shown in Eqs. (88) and (90) derived in this paper. Zaman and Baddour (2010) presented a first-order solution of particle trajectory in the combined wave-current flow. The theoretical investigations of the particle paths beneath a Stokes wave and solitary wave were recently undertaken by Constantin (2006, 2010).

This paper aims to study particle trajectories of a twodimensional wave-current field based on the fully Lagrangian framework, and to derive asymptotic solutions that can be used to describe the dynamics for the entire flow field. Previous works on progressive, standing, short-crested gravity waves and gravity-capillary waves have been summarized in the papers by Chen and Hsu (2009), Chen et al. (2010) and Hsu et al. (2010). In this paper, we look into the effect of uniform current on a gravity water wave, the motion of which is assumed to be inviscid, incompressible and irrotational. A set of governing equations in Lagrangian coordinates is derived for two-dimensional progressive gravity waves on uniform current in a constant water depth. We will construct asymptotic expansions of the solution in powers of the wave amplitude, which is assumed to be small using the Lindstedt-Poincare perturbation method. Approximate solutions including particle trajectory, Lagrangian wave period, the Lagrangian mean level and mass transport velocity are derived up to the third order. A detailed analysis of influences of the uniform current is then carried out. Finally, to validate the accuracy of the analytical results, a series of laboratory experiments are performed. The trajectories of water particles in a wave-current interaction flow are shown to have an excellent agreement with experimental data.

The problem formulation and the procedures for constructing asymptotic solutions are described in Sect. 2. In Sect. 3, we derive equations for the properties of surface-particle trajectories and present results for some selected wave-current flow. Section 4 is devoted to a description of experimental apparatus and of the experimental procedure. In Sect. 5 the trajectories of surface and subsurface particles are presented. Some concluding remarks are given in the final section.

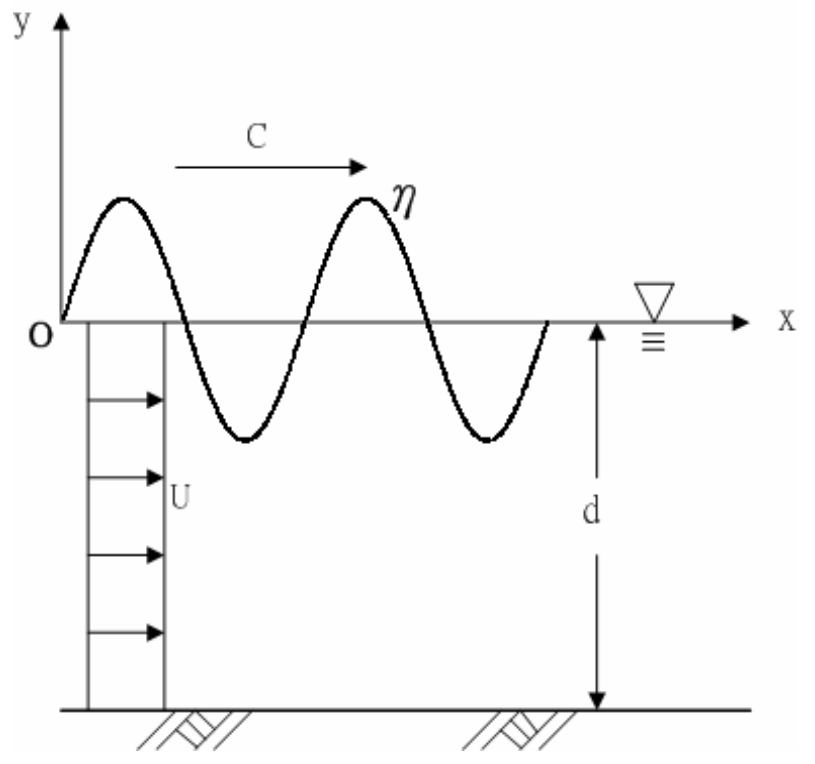

Fig. 1. Definition sketch showing a system of progressive wave train on a uniform current.

\section{Formulation of the problem}

We consider the problem of a two-dimensional monochromatic wave with a steady uniform current on an impermeable and horizontal bed (Fig. 1). The fluid motion is taken to be two-dimensional and irrotational, and the wave is rightgoing. We choose Cartesian axes with $x$ pointing horizontally to the right and $y$ vertically upward from the still water level. The mathematical problem is formulated in terms of Lagrangian variables, $a$ and $b$, which define the original position of individual fluid particles. At any time $t$, we let $b=0$ be the free surface, and $b=d$ be the bottom. The Cartesian coordinates $(x(a, b, t), y(a, b, t))$ of fluid particles and the fluid pressure $p(a, b, t)$ are the unknowns. Based on the Lagrangian description, the governing equations and boundary conditions for two-dimensional irrotational free-surface flow are summarized as follows:

$J=\frac{\partial(x, y)}{\partial(a, b)}=1$,

$$
\begin{aligned}
& \frac{\partial J}{\partial t}=x_{a t} y_{b}+x_{a} y_{b t}-x_{b t} y_{a}-x_{b} y_{a t}=0, \\
& x_{a t} x_{b}-x_{b t} x_{a}+y_{a t} y_{b}-y_{b t} y_{a}=0, \\
& \frac{\partial \phi}{\partial a}=x_{t} x_{a}+y_{t} y_{a}, \quad \frac{\partial \phi}{\partial b}=x_{t} x_{b}+y_{t} y_{b}, \\
& \frac{p}{\rho}=-\frac{\partial \phi}{\partial t}-g y+\frac{1}{2}\left[\left(\frac{\partial x}{\partial t}\right)^{2}+\left(\frac{\partial y}{\partial t}\right)^{2}\right], \\
& p=0, \quad b=0 .
\end{aligned}
$$


$v=y_{t}=0, \quad y=b=-d$.

In Eqs. (1)-(7), subscripts $a, b$, and $t$ denote partial derivatives with respect to the specified variables, $U$ denotes the speed of a steady uniform current, $g$ is the gravitational acceleration, $p(a, b, t)$ is water pressure, $\phi(a, b, t)$ is a velocity potential function in the Lagrangian system. Except for Eqs. (4) and (5), the fundamental physical relationships defining the equations above have been derived previously (Lamb, 1932; Naciri and Mei, 1992). Equation (1) is the continuity equation based on the invariant condition on the volume of a Lagrangian particle; Eq. (2) is the differentiation of Eq. (1) with respect to time. Equations (3) and (4) denote the irrotational flow condition and the corresponding Lagrangian velocity potential, respectively. Equation (5) is the Bernoulli equation for the irrotational flow in the Lagrangian description. The wave motion has to satisfy a number of boundary conditions at the bottom and on the free water surface. Equation (6) is the dynamic boundary condition of zero pressure at the free surface. On a rigid and impermeable bottom, the no-flux bottom boundary condition gives Eq. (7).

\section{Asymptotic solutions}

To solve the nonlinear Eqs. (1)-(7), we introduce the Lagrangian angular frequency $\sigma$ of particle motion, which is a function of a nonlinear parameter and the Lagrangian level label $(b)$, making the wave periodic in time $t$ and space $x$ (or $a$ ) in order to avoid a secular term. We use the LindstedtPoincare technique that yields uniform expansions to uncover the solutions in the Lagrangian system. The issue of convergence is covered by the recent regularity results in Constantin and Esher (2011). In the Lagrangian approach, the particle positions $x$ and $y$, the potential function $\phi$, and pressure $p$ are considered as functions of independent variables $a, b$ and time $t$. Following Chen and Hsu (2009), Chen et al. (2010) and Hsu et al. (2010), these solutions are sought in perturbation series by introducing an ordering parameter $\varepsilon$, which is inserted to identify the order of the associated term:

$$
\begin{aligned}
& x=a+U t+\sum_{n=1}^{\infty} \varepsilon^{n}\left[f_{n}(a, b, \sigma t)+f_{n}^{\prime}\left(a, b, \sigma_{0} t\right)\right] \\
& y=b+\sum_{n=1}^{\infty} \varepsilon^{n}\left[g_{n}(a, b, \sigma t)+g_{n}^{\prime}\left(a, b, \sigma_{0} t\right)\right] \\
& \phi=U a+\frac{1}{2} U^{2} t+\sum_{n=1}^{\infty} \varepsilon^{n}\left[\phi_{n}(a, b, \sigma t)+\phi_{n}^{\prime}\left(a, b, \sigma_{0} t\right)\right] \\
& p=-\rho g b+\sum_{n=1}^{\infty} \varepsilon^{n} p_{n}(a, b, \sigma t) \\
& \sigma=\sigma_{0}(a, b)+\sum_{n=1}^{\infty} \varepsilon^{n} \sigma_{n}(a, b)=2 \pi / T_{\mathrm{L}}
\end{aligned}
$$

where the Lagrangian variables $(a, b)$ are defined as the two characteristic parameters. In these expressions, $f_{n}, g_{n}, \phi_{n}$ and $p_{n}$ are expected to be associated with the $n$ th-order harmonic solutions. $f_{n}^{\prime}, g_{n}^{\prime}$ and $\phi_{n}^{\prime}$ are non-periodic functions that increase linearly with time. $\sigma=2 \pi / T_{\mathrm{L}}$ is the angular frequency of particle motion or the Lagrangian angular frequency for a particle reappearing at the same elevation. $T_{\mathrm{L}}$ is the corresponding period of particle motion. Upon substituting Eqs. (8)-(12) into Eqs. (1)-(7) and collecting terms of equal order, we obtain a sequence of nonhomogeneous governing equations that can be solved successively, as shown in the following sections.

\subsection{First-order approximation}

Collecting terms of order $\varepsilon$, the governing equations and the boundary conditions can be obtained as follows:

$$
\begin{aligned}
& f_{1 a}+f_{1 a}^{\prime}+g_{1 b}+g_{1 b}^{\prime}+\left[\sigma_{0 a}\left(f_{1 \sigma t}+f_{1 \sigma_{0} t}^{\prime}\right)\right. \\
& \left.+\sigma_{0 b}\left(g_{1 \sigma t}+g_{1 \sigma_{0} t}^{\prime}\right)\right] t=0, \\
& \sigma_{0}\left(f_{1 a \sigma t}+f_{1 a \sigma_{0} t}^{\prime}+g_{1 b \sigma t}+g_{1 b \sigma_{0} t}^{\prime}\right) \\
& +\sigma_{0 a}\left(f_{1 \sigma t}+f_{1 \sigma_{0} t}^{\prime}\right)+\sigma_{0 b}\left(g_{1 \sigma t}+g_{1 \sigma_{0} t}^{\prime}\right) \\
& +\sigma_{0}\left\{\sigma_{0 a}\left[f_{1(\sigma t)^{2}}+f_{1\left(\sigma_{0} t\right)^{2}}^{\prime}\right]+\sigma_{0 b}\left[g_{1(\sigma t)^{2}}+g_{1\left(\sigma_{0} t\right)^{2}}^{\prime}\right]\right\} t=0, \\
& \sigma_{0}\left(f_{1 b \sigma t}+f_{1 b \sigma_{0} t}^{\prime}-g_{1 a \sigma t}-g_{1 a \sigma_{0} t}^{\prime}\right) \\
& +\sigma_{0 b}\left(f_{1 \sigma t}+f_{1 \sigma_{0} t}^{\prime}\right)-\sigma_{0 a}\left(g_{1 \sigma t}+g_{1 \sigma_{0} t}^{\prime}\right) \\
& +\sigma_{0}\left\{\sigma_{0 b}\left[f_{1(\sigma t)^{2}}+f_{1\left(\sigma_{0} t\right)^{2}}^{\prime}\right]-\sigma_{0 a}\left[g_{1(\sigma t)^{2}}+g_{1\left(\sigma_{0} t\right)^{2}}^{\prime}\right]\right\} t=0, \\
& \phi_{1 a}+\phi_{1 a}^{\prime}+\sigma_{0 a}\left(\phi_{1 \sigma t}+\phi_{1 \sigma_{0} t}^{\prime}\right) t \\
& =U \cdot\left[\left(f_{1 a}+f_{1 a}^{\prime}\right)+\sigma_{0 a}\left(f_{1 \sigma t}+f_{1 \sigma_{0} t}^{\prime}\right) t\right]+\sigma_{0}\left(f_{1 \sigma t}+f_{1 \sigma_{0} t}^{\prime}\right), \\
& \phi_{1 b}+\phi_{1 b}^{\prime}+\sigma_{0 b}\left(\phi_{1 \sigma t}+\phi_{1 \sigma_{0} t}^{\prime}\right) t \\
& =U \cdot\left[\left(f_{1 b}+f_{1 b}^{\prime}\right)+\sigma_{0 b}\left(f_{1 \sigma t}+f_{1 \sigma_{0} t}^{\prime}\right) t\right]+\sigma_{0}\left(g_{1 \sigma t}+g_{1 \sigma_{0} t}^{\prime}\right), \\
& \frac{p_{1}}{\rho}=U \cdot \sigma_{0}\left(f_{1 \sigma t}+f_{1 \sigma_{0} t}^{\prime}\right)-\sigma_{0}\left(\phi_{1 \sigma t}+\phi_{1 \sigma_{0} t}^{\prime}\right)-g\left(g_{1}+g_{1}^{\prime}\right), \\
& p_{1}=0 \quad \text { at } \quad b=0, \\
& g_{1 \sigma t}=g_{1 \sigma_{0} t}^{\prime}=0 \quad \text { on } \quad b=-d .
\end{aligned}
$$

The flow is assumed periodic with a crest at $a=0$ and $t=0$, and hence the first-order solution can be easily written as

$$
\begin{aligned}
& f_{1}=-\alpha \frac{\cosh k(b+d)}{\cosh k d} \sin (k a-\sigma t), \\
& g_{1}=\alpha \frac{\sinh k(b+d)}{\cosh k d} \cos (k a-\sigma t), \\
& f_{1}^{\prime}=g_{1}^{\prime}=0, \\
& \sigma_{0 a}=\sigma_{0 b}=0, \\
& \phi_{1}=\left(\frac{\sigma_{0}}{k}-U\right) \alpha \frac{\cosh k(b+d)}{\cosh k d} \sin (k a-\sigma t), \\
& \sigma_{0}^{2}=g k \tanh k d,
\end{aligned}
$$




$$
\frac{p_{1}}{\rho}=-g \alpha \frac{\sinh k b}{\cosh ^{2} k d} \cos (k a-\sigma t),
$$

where the parameter $\alpha$ represents the amplitude function of the particle displacement; the wave amplitude is, as usual, taken as $a_{0}=\alpha \tanh k d$, where $k$ is the wave number $(=2 \pi / L$, $L$ is wave length). $\phi_{1}(a, b, t)$ is the first-order Lagrangian velocity potential and $p_{1}(a, b, t)$ is the first-order wave dynamic pressure in the Lagrangian form with pressure $p_{1}=0$ at the free surface $b=0$. Equations (21a-g) satisfy all the hydrodynamic equations formulated in Lagrangian terms including the irrotational condition, and differ from Gerstner's wave in infinite water depth, which possesses finite vorticity. The dispersion relation shows that the first-order Lagrangian wave frequency $\left(\sigma_{0}\right)$ is the same as that of the first-order Stokes wave frequency in the Eulerian approach (Biesel, 1952). The first-order free surface in Lagrangian coordinates is given by setting $b=0$ in Eqs. (21a) and (21b), and is similar to expressions for the profile found from the first-order Eulerian equations. Equation (21d) is the basic velocity potential solution with a steady uniform current. In Eq. (21f), $\sigma_{0}$ is the essential Lagrangian wave frequency for water particles relative to the uniform current. From this, it can be demonstrated that the Doppler's effect is not apparent in the Lagrangian dispersion relation. This is correct: in Eulerian frame of reference, intrinsic wave frequency $\sigma_{0}$ is different from absolute wave frequency $\left(\sigma_{0}-k U\right)$.

\subsection{Second-order approximation}

Collecting terms of order $\varepsilon^{2}$ and using Eq. (21), the governing equations and the boundary conditions can be obtained as

$$
\begin{aligned}
& f_{2 a}+f_{2 a}^{\prime}+g_{2 b}+g_{2 b}^{\prime}+f_{1 a} g_{1 b}-f_{1 b} g_{1 a} \\
& +\left(\sigma_{1 a} f_{1 \sigma t}+\sigma_{1 b} g_{1 \sigma t}\right) t=0 \\
& \sigma_{0}\left(f_{2 a \sigma t}+f_{2 a \sigma_{0} t}^{\prime}+g_{2 b \sigma t}+g_{2 b \sigma_{0} t}^{\prime}\right) \\
& +\sigma_{1}\left(f_{1 a}+g_{1 b}\right)_{\sigma t}+\sigma_{0}\left(f_{1 a} g_{1 b}-f_{1 b} g_{1 a}\right)_{\sigma t} \\
& +\sigma_{1 a} f_{1 \sigma t}+\sigma_{1 b} g_{1 \sigma t}+\sigma_{0}\left[\sigma_{1 a} f_{1(\sigma t)^{2}}+\sigma_{1 b} g_{1(\sigma t)^{2}}\right] t=0 \\
& \sigma_{0}\left(f_{2 b \sigma t}+f_{2 b \sigma_{0} t}^{\prime}-g_{2 a \sigma t}-g_{2 a \sigma_{0} t}^{\prime}\right) \\
& +\sigma_{1}\left(f_{1 b}-g_{1 a}\right)_{\sigma t}+\sigma_{1 b} f_{1 \sigma t}-\sigma_{1 a} g_{1 \sigma t} \\
& +\sigma_{0}\left(f_{1 a} f_{1 b \sigma t}-f_{1 a \sigma t} f_{1 b}+g_{1 a} g_{1 b \sigma t}-g_{1 b} g_{1 a \sigma t}\right) \\
& +\sigma_{0}\left[\sigma_{1 b} f_{1(\sigma t)^{2}}-\sigma_{1 a} g_{1(\sigma t)^{2}}\right] t=0 \\
& \phi_{2 a}+\phi_{2 a}^{\prime}=U \cdot\left[\left(f_{2 a}+f_{2 a}^{\prime}\right)\right. \\
& \left.+\sigma_{0 a}\left(f_{2 \sigma t}+f_{2 \sigma_{0} t}^{\prime}\right) t\right]+U \cdot \sigma_{1 a} t f_{1 \sigma t} \\
& -\sigma_{0 a}\left(\phi_{2 \sigma t}+\phi_{2 \sigma_{0} t}^{\prime}\right) \\
& +\sigma_{0}\left(f_{2 \sigma t}+f_{2 \sigma_{0} t}^{\prime}\right)+\sigma_{1} f_{1 \sigma t} \\
& +\sigma_{0}\left(f_{1 a} f_{1 \sigma t}+g_{1 a} g_{1 \sigma t}\right)-\sigma_{1 a} t \phi_{1 \sigma t} \\
& \phi_{2 b}+\phi_{2 b}^{\prime}=U \cdot\left[\left(f_{2 b}+f_{2 b}^{\prime}\right)+\sigma_{0 b}\left(f_{2 \sigma t}+f_{2 \sigma_{0} t}^{\prime}\right) t\right] \\
& +U \cdot \sigma_{1 b} t f_{1 \sigma t}-\sigma_{0 b}\left(\phi_{2 \sigma t}+\phi_{2 \sigma_{0} t}^{\prime}\right) \\
& +\sigma_{0}\left(g_{2 \sigma t}+g_{2 \sigma_{0} t}^{\prime}\right)+\sigma_{1} g_{1 \sigma t} \\
& +\sigma_{0}\left(f_{1 b} f_{1 \sigma t}+g_{1 b} g_{1 \sigma t}\right)-\sigma_{1 b} t \phi_{1 \sigma t} \\
&
\end{aligned}
$$

$$
\begin{aligned}
& \frac{p_{2}}{\rho}=-\left[\sigma_{0}\left(\phi_{2 \sigma t}+\phi_{2 \sigma_{0} t}^{\prime}\right)+g\left(g_{2}+g_{2}^{\prime}\right)\right] \\
& -\sigma_{1} \phi_{1 \sigma t}+\frac{1}{2} \sigma_{0}^{2}\left(f_{1 \sigma t}^{2}+g_{1 \sigma t}^{2}\right) \\
& +U \cdot \sigma_{0}\left(f_{2 \sigma t}+f_{2 \sigma_{0} t}^{\prime}\right)+U \cdot \sigma_{1}\left(f_{1 \sigma t}+f_{1 \sigma_{0} t}^{\prime}\right),
\end{aligned}
$$

and

$p_{2}=0 \quad$ at $\quad b=0$.

$g_{2 \sigma t}=g_{2 \sigma_{0} t}^{\prime}=0$ on $\quad b=-d$.

Substituting Eqs. (21a $\mathrm{g}$ ) into Eqs. (22)-(24), the secondorder governing equations in terms of $\varepsilon^{2}$, including the continuity equation and the irrotational condition, are given by

$$
\begin{aligned}
& f_{2 a}+f_{2 a}^{\prime}+g_{2 b}+g_{2 b}^{\prime} \\
& =\frac{1}{2} k^{2} \alpha^{2} \cdot\left\{\frac{\cosh 2 k(b+d)}{\cosh ^{2} k d}+\frac{\cos 2(k a-\sigma t)}{\cosh ^{2} k d}\right\} \\
& -\alpha \cdot\left\{\sigma_{1 a} \cdot \frac{\cosh k(b+d)}{\cosh k d} \cos (k a-\sigma t)\right. \\
& \left.+\sigma_{1 b} \cdot \frac{\sinh k(b+d)}{\cosh k d} \sin (k a-\sigma t)\right\} \cdot t, \\
& \sigma_{0}\left(f_{2 a \sigma t}+f_{2 a \sigma_{0} t}^{\prime}+g_{2 b \sigma t}+g_{2 b \sigma_{0} t}^{\prime}\right) \\
& =\alpha^{2} \cdot k^{2} \cdot \sigma_{0} \cdot \frac{\sin 2(k a-\sigma t)}{\cosh ^{2} k d} \\
& -\alpha \cdot\left[\sigma_{1 a} \frac{\cosh k(b+d)}{\cosh k d} \cos (k a-\sigma t)\right. \\
& \left.+\sigma_{1 b} \frac{\sinh k(b+d)}{\cosh k d} \sin (k a-\sigma t)\right] \\
& -\alpha \cdot \sigma_{0} \cdot\left[\sigma_{1 a} \frac{\cosh k(b+d)}{\cosh k d} \sin (k a-\sigma t)\right. \\
& \left.-\sigma_{1 b} \frac{\sinh k(b+d)}{\cosh k d} \cos (k a-\sigma t)\right] t, \\
& \sigma_{0}\left(f_{2 b \sigma t}+f_{2 b \sigma_{0} t}^{\prime}-g_{2 a \sigma t}-g_{2 a \sigma_{0} t}^{\prime}\right) \\
& =\alpha^{2} k^{2} \sigma_{0} \frac{\sinh 2 k(b+d)}{\cosh { }^{2} k d} \\
& -\alpha \cdot\left[\sigma_{1 b} \frac{\cosh k(b+d)}{\cosh k d} \cos (k a-\sigma t)\right. \\
& \left.-\sigma_{1 a} \frac{\sinh k(b+d)}{\cosh k d} \sin (k a-\sigma t)\right] \\
& -\alpha \cdot \sigma_{0} \cdot\left[\sigma_{1 a} \frac{\sinh k(b+d)}{\cosh k d} \cos (k a-\sigma t)\right. \\
& \left.+\sigma_{1 b} \frac{\cosh k(b+d)}{\cosh k d} \sin (k a-\sigma t)\right] t .
\end{aligned}
$$

For gravity waves of permanent form, the terms $t \cos (k a-$ $\sigma t)$ and $t \sin (k a-\sigma t)$ that increase linearly with time have to be zero to avoid resonance. Noting that $\sigma_{1 b}=0$ or $\sigma_{1}=\omega_{1}=$ constant, then the general solution that satisfies the bottom boundary condition can be written as

$$
\begin{aligned}
& f_{2}=-\beta_{2} \frac{\cosh 2 k(b+d)}{\cosh ^{2} k d} \sin 2(k a-\sigma t) \\
& +\frac{1}{4} \alpha^{2} k \frac{\sin 2(k a-\sigma t)}{\cosh ^{2} k d} \\
& -\lambda_{2} \frac{\cosh k(b+d)}{\cosh k d} \sin (k a-\sigma t) \\
& f_{2}^{\prime}=\frac{1}{2} \alpha^{2} k \frac{\cosh 2 k(b+d)}{\cosh ^{2} k d} \sigma_{0} t \\
& g_{2}=\beta_{2} \frac{\sinh 2 k(b+d)}{\cosh ^{2} k d} \cos 2(k a-\sigma t) \\
& +\frac{1}{4} \alpha^{2} k \frac{\sinh 2 k(b+d)}{\cosh ^{2} k d}+\lambda_{2} \frac{\sinh k(b+d)}{\cosh k d} \cos (k a-\sigma t)
\end{aligned}
$$


$g_{2}^{\prime}=0$.

Substituting Eqs. (33)-(36) into the irrotational Eq. (25) in $\varepsilon^{2}$ order, we obtain

$$
\begin{aligned}
& \phi_{2 a}=-2 k U \beta_{2} \frac{\cosh 2 k(b+d)}{\cosh ^{2} k d} \cos 2(k a-\sigma t) \\
& +\frac{1}{2} \alpha^{2} k^{2} U \frac{1}{\cosh ^{2} k d} \cos 2(k a-\sigma t) \\
& -\lambda_{2} k U \frac{\cosh k(b+d)}{\cosh k d} \cos (k a-\sigma t) \\
& +\sigma_{0}\left[2 \beta_{2} \frac{\cosh 2 k(b+d)}{\cosh ^{2} k d} \cos 2(k a-\sigma t)\right. \\
& \left.-\alpha^{2} k \frac{\cos 2(k a-\sigma t)}{\cosh ^{2} k d}\right] \\
& +\left(\alpha \sigma_{1}+\sigma_{0} \lambda_{2}\right) \frac{\cosh k(b+d)}{\cosh k d} \cos (k a-\sigma t)
\end{aligned}
$$

$\phi_{2 b}+\phi_{2 b}^{\prime}=-2 k U \beta_{2} \frac{\sinh 2 k(b+d)}{\cosh ^{2} k d} \sin 2(k a-\sigma t)$

$-k U \lambda_{2} \frac{\sinh k(b+d)}{\cosh k d} \sin (k a-\sigma t)$

$+\alpha^{2} k^{2} U \frac{\sinh 2 k(b+d)}{\cosh ^{2} k d} \sigma_{0} t$

$+2 \sigma_{0} \beta_{2} \frac{\sinh 2 k(b+d)}{\cosh ^{2} k d} \sin 2(k a-\sigma t)$

$+\sigma_{0} \lambda_{2} \frac{\sinh k(b+d)}{\cosh k d} \sin (k a-\sigma t)$

$+\sigma_{1} \alpha \frac{\sinh k(b+d)}{\cosh k d} \sin (k a-\sigma t)$.

Note that the secular terms in Eqs. (37) and Sect. 3.2 have to be eliminated. The second-order Lagrangian velocity potential is obtained by integrating over the Lagrangian variables $a$ or $b$ as

$$
\begin{aligned}
& \phi_{2}=\frac{\sigma_{0}-k U}{k} \beta_{2} \frac{\cosh 2 k(b+d)}{\cosh ^{2} k d} \sin 2(k a-\sigma t) \\
& -\frac{1}{2} \alpha^{2}\left(\sigma_{0}-\frac{1}{2} k U\right) \frac{1}{\cosh ^{2} k d} \sin 2(k a-\sigma t) \\
& +\frac{1}{2} \alpha^{2} k U \frac{\cosh 2 k(b+d)}{\cosh ^{2} k d} \sigma_{0} t+D_{2}\left(\sigma_{0} t\right) .
\end{aligned}
$$

and

$\alpha \omega_{1}+\lambda_{2}\left(\sigma_{0}-k U\right)=0$.

Substituting the solutions up to the second order into the energy Eq. (27) in $\varepsilon^{2}$ order, we can get

$$
\begin{aligned}
& \frac{p_{2}}{\rho}=\left\{2 \sigma_{0} \frac{\sigma_{0}-k U}{k} \beta_{2} \frac{\cosh 2 k(b+d)}{\cosh ^{2} k d}-\frac{3}{4} \sigma_{0}^{2} \alpha^{2} \frac{1}{\cosh ^{2} k d}\right. \\
& \left.-g \beta_{2} \frac{\sinh 2 k(b+d)}{\cosh ^{2} k d}+2 U \sigma_{0} \beta_{2} \frac{\cosh 2 k(b+d)}{\cosh ^{2} k d}\right\} \cdot \cos 2(k a-\sigma t) \\
& +\left\{-g \lambda_{2} \frac{\sinh ^{2} k(b+d)}{\cosh k d}+\sigma_{1} \frac{\sigma_{0}-k U}{k} \alpha \frac{\cosh ^{2} k(b+d)}{\cosh k d}\right. \\
& \left.+U \sigma_{0} \lambda_{2} \frac{\cosh k(b+d)}{\cosh k d}+U \sigma_{1} \alpha \frac{\cosh k(b+d)}{\operatorname{coshh} k d}\right\} \cdot \cos (k a-\sigma t) \\
& +\left\{-\sigma_{0} D_{2 \sigma_{0} t}-\frac{1}{4} g \alpha^{2} k \frac{\sinh 2 k(b+d)}{\cosh ^{2} k d}+\frac{1}{4} \sigma_{0}^{2} \alpha^{2} \frac{\cosh 2 k(b+d)}{\cosh ^{2} k d}\right\} .
\end{aligned}
$$

Applying the zero pressure condition at the free surface, the unknown coefficients in Eq. (41) are obtained as

$$
\begin{aligned}
& \lambda_{2}=0, \quad \omega_{1}=0, \beta_{2}=\frac{3}{8} \alpha^{2} k\left(\tanh ^{-2} k d-1\right), \\
& D_{2}=\phi_{2}^{\prime}\left(\sigma_{0} t\right)=\frac{1}{4} \alpha^{2} \sigma_{0}^{2}\left(\tanh ^{2} k d-1\right) t .
\end{aligned}
$$

The second-order Lagrangian solutions are assembled as

$$
\begin{aligned}
& f_{2}=-\frac{3}{8} \alpha^{2} k\left(\tanh ^{-2} k d-\tanh ^{2} k d\right) \frac{\cosh 2 k(b+d)}{\cosh 2 k d} \sin 2(k a-\sigma t) \\
& +\frac{1}{4} \alpha^{2} k\left(1-\tanh ^{2} k d\right) \sin 2(k a-\sigma t), \\
& f_{2}^{\prime}=\frac{1}{2} \alpha^{2} k\left(1+\tanh ^{2} k d\right) \frac{\cosh 2 k(b+d)}{\cosh 2 k d} \sigma_{0} t, \\
& g_{2}=\frac{3}{8} \alpha^{2} k\left(\tanh ^{-2} k d-\tanh ^{2} k d\right) \frac{\sinh 2 k(b+d)}{\operatorname{coshh} 2 k d} \\
& \cos 2(k a-\sigma t)+\frac{1}{4} \alpha^{2} k\left(1+\tanh ^{2} k d\right) \frac{\sinh 2 k(b+d)}{\cosh 2 k d} \\
& g_{2}^{\prime}=\sigma_{1}=0, \\
& \phi_{2}=\frac{\sigma_{0}-k U}{k} \beta_{2} \frac{\cosh 2 k(b+d)}{\cosh ^{2} k d} \sin 2(k a-\sigma t) \\
& -\frac{1}{2} \alpha^{2}\left(\sigma_{0}-k U\right) \frac{1}{\cosh ^{2} k d} \sin 2(k a-\sigma t) \\
& -\frac{1}{4} \alpha^{2} k U \frac{1}{\cosh ^{2} k d} \sin ^{2}(k a-\sigma t), \\
& \phi_{2}^{\prime}=\frac{1}{4} \alpha^{2} \sigma_{0}^{2}\left(\tanh ^{2} k d-1\right) t+\frac{1}{2} \alpha^{2} k U \frac{\cosh ^{2} k(b+d)}{\cosh ^{2} k d} \sigma_{0} t \\
& \frac{p_{2}}{\rho}=\left\{2 \sigma_{0} \frac{\sigma_{0}-k U}{k} \beta_{2} \frac{\cosh ^{2} 2 k(b+d)}{\cosh ^{2} k d}\right. \\
& -g \beta_{2} \frac{\sinh _{2} k(b+d)}{\cosh ^{2} k d}-\frac{3}{4} \sigma_{0}^{2} \alpha^{2} \frac{1}{\cosh ^{2} k d} \\
& \left.+2 U \sigma_{0} \beta_{2}\right\} \cdot \cos _{2} 2(k a-\sigma t) \\
& -\frac{1}{4} \alpha^{2} \sigma_{0}^{2}\left(\tanh ^{2} k d-1\right)-\frac{1}{4} g \alpha^{2} k \frac{\sinh ^{2} k(b+d)}{\cosh ^{2} k d} \\
& +\frac{1}{4} \alpha^{2} \sigma_{0}^{2} \frac{\cosh ^{2} k(b+d)}{\cosh ^{2} k d} .
\end{aligned}
$$

The Lagrangian formulation for the particle trajectory at the second order approximation comprises a periodic component $f_{2}$ and non-periodic function $f_{2}^{\prime}$. The latter increases linearly with time and is independent of the Lagrangian horizontal label $a$, which represents the mass transport, implying that a constant net motion would depend only on the vertical level $b$ where the particle is located in the uniform current. The trajectory is the smallest near the bottom and is not a closed orbit as predicted by the first-order approximation. Moreover, Eq. (33), a second-order quantity, renders the same form obtained by Longuet-Higgins (1953) for the case of wave alone (i.e., without uniform current). The solution for vertical displacement $g_{2}$ includes a second harmonic component and a time-independent term which is a function of wave steepness and the Lagrangian vertical label $b$. Overall, the expression of $g_{2}$ yields vertical shift correction to a second-order which decreases with water depth. Taking the time average of the particle elevation $g_{2}$ over a given period of a particle motion from Eq. (35), it can be shown that the mean level of water particle orbit in Lagrangian approach is higher than 
that in the Eulerian counterpart, as suggested by LonguetHiggins $(1979,1986)$ for two-dimensional progressive water waves.

For the limiting case $U=0$, one can verify that the present theory reduces to pure progressive waves of constant depth, as was previously obtained by Chen et al. (2010).

\subsection{Third-order approximation}

The third-order governing equations and boundary conditions can be obtained by collecting the terms of

$$
\begin{aligned}
& f_{3 a}+f_{3 a}^{\prime}+g_{3 b}+g_{3 b}^{\prime}+f_{1 a} g_{2 b}+f_{2 a} g_{1 b}-f_{1 b} g_{2 a} \\
& -\left(f_{2 b}+f_{2 b}^{\prime}\right) g_{1 a}+\left(\sigma_{2 a} f_{1 \sigma t}+\sigma_{2 b} g_{1 \sigma t}\right) t=0, \\
& \sigma_{0}\left(f_{3 a \sigma t}+f_{3 a \sigma_{0} t}^{\prime}+g_{3 b \sigma t}+g_{3 b \sigma_{0} t}^{\prime}\right)+\sigma_{2}\left(f_{1 a \sigma t}+g_{1 b \sigma t}\right) \\
& +\sigma_{2 a} f_{1 \sigma t}+\sigma_{2 b} g_{1 \sigma t}+\sigma_{0}\left[\sigma_{2 a} f_{1(\sigma t)^{2}}\right. \\
& \left.+\sigma_{2 b} g_{1(\sigma t)^{2}}\right]+\sigma_{0}\left[f_{1 a \sigma t} g_{2 b}+f_{1 a} g_{2 b \sigma t}\right. \\
& +f_{2 a \sigma t} g_{1 b}+f_{2 a} g_{1 b \sigma t}-f_{1 b \sigma t} g_{2 a}-f_{1 b} g_{2 a \sigma t}-\left(f_{2 b \sigma t}\right. \\
& \left.\left.+f_{2 b \sigma_{0}}^{\prime}\right) g_{1 a}-\left(f_{2 b}+f_{2 b}^{\prime}\right) g_{1 a \sigma t}\right]=0, \\
& \sigma_{0}\left(f_{3 b \sigma t}+f_{3 b \sigma_{0} t}^{\prime}-g_{3 a \sigma t}-g_{3 a \sigma_{0} t}^{\prime}\right)+\sigma_{2}\left(f_{1 b \sigma t}-g_{1 a \sigma t}\right) \\
& +\sigma_{2 b} f_{1 \sigma t}-\sigma_{2 a} g_{1 \sigma t}+\sigma_{0}\left[\sigma_{2 b} f_{1(\sigma t)^{2}}\right. \\
& \left.-\sigma_{2 a} g_{1(\sigma t)^{2}}\right] t+\sigma_{0}\left[f_{1 a}\left(f_{2 b \sigma t}+f_{2 b \sigma_{0} t}^{\prime}\right)\right. \\
& +f_{2 a} f_{1 b \sigma t}-f_{2 a \sigma t} f_{1 b} \\
& -f_{1 a \sigma t}\left(f_{2 b}+f_{2 b}^{\prime}\right)+g_{1 a} g_{2 b \sigma t}+g_{2 a} g_{1 b \sigma t}-g_{1 b} g_{2 a \sigma t} \\
& \left.-g_{2 b} g_{1 a \sigma t}\right]=0, \\
& \phi_{3 a}+\phi_{3 a}^{\prime}=U \cdot\left[\left(f_{3 a}+f_{3 a}^{\prime}\right)+\sigma_{0 a}\left(f_{3 \sigma t}+f_{3 \sigma_{0} t}^{\prime}\right) t\right] \\
& +\sigma_{0}\left(f_{3 \sigma t}+f_{3 \sigma_{0} t}^{\prime}\right)+\sigma_{2} f_{1 \sigma t}+\sigma_{0}\left[f_{1 \sigma t} f_{2 a}\right. \\
& \left.+\left(f_{2 \sigma t}+f_{2 \sigma_{0} t}^{\prime}\right) f_{1 a}+g_{1 \sigma t} g_{2 a}+g_{2 \sigma t} g_{1 a}\right]-\sigma_{2 a} t \phi_{1 \sigma t}, \\
& \phi_{3 b}+\phi_{3 b}^{\prime}=U \cdot\left[\left(f_{3 b}+f_{3 b}^{\prime}\right)\right. \\
& \left.+\sigma_{0 b}\left(f_{3 \sigma t}+f_{3 \sigma_{0} t}^{\prime}\right) t\right]+\left(U f_{1 \sigma t}\right. \\
& \left.-\phi_{1 \sigma t}\right) \sigma_{2 b} t+\sigma_{0}\left(g_{3 \sigma t}+g_{3 \sigma_{0} t}^{\prime}\right) \\
& +\sigma_{2} g_{1 \sigma t}+\sigma_{0}\left[f_{1 \sigma t}\left(f_{2 b}+f_{2 b}^{\prime}\right)\right. \\
& \left.+\left(f_{2 \sigma t}+f_{2 \sigma_{0} t}^{\prime}\right) f_{1 b}+g_{1 \sigma t} g_{2 b}+g_{2 \sigma t} g_{1 b}\right], \\
& \frac{p_{3}}{\rho}=U \cdot \sigma_{0}\left(f_{3 \sigma t}\right. \\
& \left.+f_{3 \sigma_{0} t}^{\prime}\right)+U \cdot \sigma_{1} f_{2 \sigma t}+ \\
& U \cdot \sigma_{2} f_{1 \sigma t}-\left[\sigma _ { 0 } \left(\phi_{3 \sigma t}\right.\right. \\
& \left.\left.+\phi_{3 \sigma_{0} t}^{\prime}\right)+g\left(g_{3}+g_{3}^{\prime}\right)\right] \\
& -\sigma_{2} \phi_{1 \sigma t}+\sigma_{0}^{2}\left[f_{1 \sigma t}\left(f_{2 \sigma t}+f_{2 \sigma_{0} t}^{\prime}\right)\right. \\
& \left.+g_{1 \sigma t} g_{2 \sigma t}\right], \\
& p_{3}=0 \quad \text { at } \quad b=0, \\
& g_{3 \sigma t}=g_{3 \sigma_{0} t}^{\prime}=0 \quad \text { on } \quad b=-d . \\
& O n
\end{aligned}
$$

On substituting the first- and second-order approximations into the governing Eqs. (50)-(52), the third-order continuity, irrotational and energy equations become

$$
\begin{aligned}
& f_{3 a}+f_{3 a}^{\prime}+g_{3 b}+g_{3 b}^{\prime} \\
& =\alpha k^{2}\left(2 \beta_{2}+\frac{1}{4} \alpha^{2} k\right) \frac{\cosh _{3} k(b+d)}{\cosh ^{3} k d} \cos (k a-\sigma t) \\
& +\alpha k^{2}\left(2 \beta_{2}-\frac{1}{4} \alpha^{2} k\right) \frac{\cosh k(b+d)^{\cosh ^{3} k d} \cos 3(k a-\sigma t)}{\cos (k)} \\
& -\alpha \cdot\left\{\left[\alpha^{2} k^{3} \sigma_{0} \frac{\sinh 2 k(b+d)}{\cosh ^{2} k d}+\sigma_{2 b}\right] \cdot \frac{\sinh k(b+d)}{\cosh k d} \sin (k a-\sigma t)\right. \\
& \left.+\sigma_{2 a} \frac{\cosh k(b+d)}{\cosh k d} \cos (k a-\sigma t)\right\} \cdot t,
\end{aligned}
$$

$$
\begin{aligned}
& \sigma_{0}\left(f_{3 a \sigma t}+f_{3 a \sigma_{0} t}^{\prime}+g_{3 b \sigma t}+g_{3 b \sigma_{0} t}^{\prime}\right) \\
& =\alpha k^{2} \sigma_{0}\left[\left(2 \beta_{2}+\frac{1}{4} \alpha^{2} k\right) \frac{\cosh 3 k(b+d)}{\cosh ^{3} k d} \sin (k a-\sigma t)\right. \\
& \left.+\left(6 \beta_{2}-\frac{3}{4} \alpha^{2} k\right) \frac{\cosh k(b+d)}{\cosh ^{3} k d} \sin 3(k a-\sigma t)\right] \\
& -\alpha \cdot\left\{\left[\alpha^{2} k^{3} \sigma_{0} \frac{\sinh ^{2} k \cosh ^{3} k d}{\cosh ^{2} k d}+\sigma_{2 b}\right] \frac{\sinh k(b+d)}{\cosh k d} \sin (k a-\sigma t)\right. \\
& \left.+\sigma_{2 a} \frac{\cosh k(b+d)}{\cosh k d} \cos (k a-\sigma t)\right\} \\
& +\alpha \cdot \sigma_{0}\left\{\left[\alpha^{2} k^{3} \sigma_{0} \frac{\sinh 2 k(b+d)}{\cosh ^{2} k d}+\sigma_{2 b}\right] \frac{\sinh k(b+d)}{\cosh k d} \cos (k a-\sigma t)\right. \\
& \left.-\sigma_{2 a} \frac{\cosh k(b+d)}{\cosh k d} \sin (k a-\sigma t)\right\} \cdot t \text {, } \\
& \sigma_{0}\left(f_{3 b \sigma t}+f_{3 b \sigma_{0} t}^{\prime}-g_{3 a \sigma t}-g_{3 a \sigma_{0} t}^{\prime}\right) \\
& =\alpha k^{2} \sigma_{0}\left[\left(6 \beta_{2}+\frac{3}{4} \alpha^{2} k\right) \frac{\sinh 3 k(b+d)}{\cosh ^{3} k d} \cos (k a-\sigma t)\right. \\
& \left.+\left(2 \beta_{2}+\frac{1}{4} \alpha^{2} k\right) \frac{\sinh k(b+d)}{\cosh ^{3} k d} \cos 3(k a-\sigma t)\right] \\
& -\alpha \cdot\left\{\left[\frac{1}{2} \alpha^{2} k^{3} \sigma_{0} \frac{\sinh ^{2}(b+d)}{\cosh ^{3} k d}+\sigma_{2 b} \frac{\cosh k(b+d)}{\cosh k d}\right] \cos (k a-\sigma t)\right. \\
& \left.-\sigma_{2 a} \frac{\sinh k(b+d)}{\cosh k d} \sin (k a-\sigma t)\right\} \\
& -\alpha \cdot \sigma_{0}\left\{\left[\alpha^{2} k^{3} \sigma_{0} \frac{\sinh 2 k(b+d)}{\cosh ^{2} k d}\right.\right. \\
& \left.+\sigma_{2 b}\right] \frac{\cosh k(b+d)}{\cosh k d} \sin (k a-\sigma t) \\
& \left.+\sigma_{2 a} \frac{\sinh k(b+d)}{\cosh k d} \cos (k a-\sigma t)\right\} \cdot t .
\end{aligned}
$$

From Eqs. (58)-(60), the secular terms that grow with time have to be zero. We can obtain

$\sigma_{2 a}=0$ and $\sigma_{2 b}=-\alpha^{2} k^{3} \sigma_{0} \frac{\sinh 2 k(b+d)}{\cosh ^{2} k d}$.

Integrating Eq. (61) with $b, \sigma_{2}$ is given by

$$
\sigma_{2}=-\frac{1}{2} \alpha^{2} k^{2} \sigma_{0} \frac{\cosh 2 k(b+d)}{\cosh ^{2} k d}+\omega_{2},
$$

where $\omega_{2}$ is a constant which needs to be solved.

Using Eq. (62), Eqs. (58)-(60) can be reduced to

$$
\begin{aligned}
& f_{3 a}+f_{3 a}^{\prime}+g_{3 b}+g_{3 b}^{\prime}=\alpha k^{2}\left(2 \beta_{2}\right. \\
& \left.+\frac{1}{4} \alpha^{2} k\right) \frac{\cosh 3 k(b+d)}{\cosh ^{3} k d} \cos (k a-\sigma t) \\
& +\alpha k^{2}\left(2 \beta_{2}-\frac{1}{4} \alpha^{2} k\right) \frac{\cosh k(b+d)}{\cosh ^{3} k d} \cos 3(k a-\sigma t), \\
& \sigma_{0}\left(f_{3 a \sigma t}+f_{3 a \sigma_{0} t}^{\prime}+g_{3 b \sigma t}+g_{3 b \sigma_{0} t}^{\prime}\right) \\
& =\alpha k^{2} \sigma_{0}\left[\left(2 \beta_{2}+\frac{1}{4} \alpha^{2} k\right) \frac{\cosh ^{2} k(b+d)}{\cosh ^{3} k d} \sin (k a-\sigma t)\right. \\
& \left.+\left(6 \beta_{2}-\frac{3}{4} \alpha^{2} k\right) \frac{\cosh ^{2} k(b+d)}{\cosh ^{3} k d} \sin ^{2} 3(k a-\sigma t)\right], \\
& \sigma_{0}\left(f_{3 b \sigma t}+f_{3 b \sigma_{0} t}^{\prime}-g_{3 a \sigma t}-g_{3 a \sigma_{0} t}^{\prime}\right) \\
& =\alpha k^{2} \sigma_{0}\left[\left(6 \beta_{2}+\frac{5}{4} \alpha^{2} k\right) \frac{\sinh 3 k(b+d)}{\cosh ^{3} k d} \cos (k a-\sigma t)\right. \\
& \left.+\left(2 \beta_{2}+\frac{1}{4} \alpha^{2} k\right) \frac{\sinh ^{2}(b+d)}{\cosh ^{3} k d} \cos ^{3} 3(k a-\sigma t)\right] .
\end{aligned}
$$

From Eqs. (63)-(65), the solutions of $f_{3}, f_{3}^{\prime}, g_{3}$ and $g_{3}^{\prime}$ can be assumed as

$$
\begin{aligned}
& f_{3}=\left[-\beta_{3} \frac{\cosh 3 k(b+d)}{\cosh ^{3} k d}+\frac{1}{6} \alpha k\left(5 \beta_{2}\right.\right. \\
& \left.\left.-\frac{1}{2} \alpha^{2} k\right) \frac{\cosh ^{2}(b+d)}{\cosh ^{3} k d}\right] \sin 3(k a-\sigma t) \\
& -\left[\frac{1}{2} \alpha k\left(5 \beta_{2}+\alpha^{2} k\right) \frac{\cosh 3 k(b+d)}{\cosh ^{3} k d}\right. \\
& \left.+\lambda_{3} \frac{\cosh k(b+d)}{\cosh ^{3} k d}\right] \sin (k a-\sigma t), \\
& g_{3}=\left[\beta_{3} \frac{\sinh 3 k(b+d)}{\cosh ^{3} k d}-\frac{1}{2} \alpha k \beta_{2} \frac{\sinh k(b+d)}{\cosh ^{3} k d}\right] \cos 3(k a-\sigma t) \\
& +\left[\frac{1}{2} \alpha k\left(3 \beta_{2}+\frac{1}{2} \alpha^{2} k\right) \frac{\sinh 3 k(b+d)}{\cosh ^{3} k d}\right. \\
& \left.+\lambda_{3} \frac{\sinh ^{2}(b+d)}{\cosh ^{3} k d}\right] \cos (k a-\sigma t),
\end{aligned}
$$


$f_{3}^{\prime}=g_{3}^{\prime}=0$

where $\beta_{3}$ and $\lambda_{3}$ are undetermined coefficients which can be found by using the dynamic free surface boundary condition.

Substituting these terms, the first- and the second-order solutions into Eqs. (53) and (54), we can get

$$
\begin{aligned}
& \phi_{3 a}+\phi_{3 a}^{\prime}=U \cdot\left[-3 k \beta_{3} \frac{\cosh 3 k(b+d)}{\cosh ^{3} k d}\right. \\
& \left.+\frac{1}{2} \alpha k^{2}\left(5 \beta_{2}-\frac{1}{2} \alpha^{2} k\right) \frac{\cosh k(b+d)}{\cosh ^{3} k d}\right] \cos 3(k a-\sigma t) \\
& -U \cdot\left[\frac{1}{2} \alpha k^{2}\left(5 \beta_{2}+\alpha^{2} k\right) \frac{\cosh 3 k(b+d)}{\cosh ^{3} k d}\right. \\
& \left.+k \lambda_{3} \frac{\cosh k(b+d)}{\cosh ^{3} k d}\right] \cos (k a-\sigma t) \\
& +3 \sigma_{0} \cdot\left[\beta_{3} \frac{\cosh ^{2} k(b+d)}{\cosh ^{3} k d}-\frac{1}{2} \alpha k\left(3 \beta_{2}-\frac{1}{2} \alpha^{2} k\right) \frac{\cosh k(b+d)}{\cosh ^{3} k d}\right] \\
& \cdot \cos 3(k a-\sigma t) \\
& +\sigma_{0} \cdot\left[\frac{1}{2} \alpha k \beta_{2} \frac{\cosh 3 k(b+d)}{\cosh ^{3} k d}+\left(\alpha \cdot \frac{\omega_{2}}{\sigma_{0}}\right.\right. \\
& \left.\left.+\lambda_{3} \cdot \operatorname{sech}^{2} k d\right) \frac{\cosh k(b+d)}{\cosh ^{3} k d}\right] \cdot \cos (k a-\sigma t), \\
& \phi_{3 b}+\phi_{3 b}^{\prime}=U \cdot\left[-3 k \beta_{3} \frac{\sinh 3 k(b+d)}{\cosh ^{3} k d}\right. \\
& \left.+\frac{1}{6} \alpha k^{2}\left(5 \beta_{2}-\frac{1}{2} \alpha^{2} k\right) \frac{\sinh k(b+d)}{\cosh ^{3} k d}\right] \sin 3(k a-\sigma t) \\
& -U \cdot\left[\frac{3}{2} \alpha k^{2}\left(5 \beta_{2}+\alpha^{2} k\right) \frac{\sinh ^{\cos k(b+d)}}{\cosh ^{3} k d}\right. \\
& \left.+k \lambda_{3} \frac{\sinh k(b+d)}{\cosh ^{3} k d}\right] \sin (k a-\sigma t) \\
& +3 \sigma_{0} \cdot\left[\beta_{3} \sin 3(k a-\sigma t)\right. \\
& \left.+\frac{1}{2} \alpha k \beta_{2} \sin (k a-\sigma t)\right] \cdot \frac{\sin 3 k(b+d)}{\cosh ^{3} k d} \\
& +\sigma_{0} \cdot\left[-\frac{1}{2} \alpha k\left(3 \beta_{2}-\frac{1}{2} \alpha^{2} k\right) \sin 3(k a-\sigma t)\right. \\
& \left.+\left(\alpha \cdot \frac{\omega_{2}}{\sigma_{0}} \cosh ^{2} k d+\lambda_{3}\right) \sin (k a-\sigma t)\right] \frac{\sinh k(b+d)}{\cosh ^{3} k d},
\end{aligned}
$$

From Eqs. (69) and (70), we get

$$
\begin{aligned}
& \phi_{3}=U \cdot\left[-\beta_{3} \frac{\cosh ^{3} k(b+d)}{\cosh ^{3} k d}+\frac{1}{6} \alpha k\left(5 \beta_{2}-\frac{1}{2} \alpha^{2} k\right) \frac{\cosh ^{2}(b+d)}{\cosh ^{3} k d}\right] \\
& \cdot \sin 3(k a-\sigma t)-U \cdot\left[\frac{1}{2} \alpha k\left(5 \beta_{2}+\alpha^{2} k\right) \frac{\cosh ^{3} k(b+d)}{\cosh ^{3} k d}+\right. \\
& \left.k \lambda_{3} \frac{\cosh k(b+d)}{\cosh ^{3} k d}\right] \cdot \sin (k a-\sigma t) \\
& +\frac{\sigma_{0}}{k} \beta_{3} \frac{\cosh ^{2} k(b+d)}{\cosh ^{3} k d} \sin 3(k a-\sigma t) \\
& -\frac{1}{2} \alpha\left(3 \beta_{2}-\frac{1}{2} \alpha^{2} k\right) \sigma_{0} \frac{\cosh ^{2}(b+d)}{\cosh ^{3} k d} \sin 3(k a-\sigma t) \\
& +\frac{1}{2} \alpha \beta_{2} \sigma_{0} \\
& \frac{\cosh ^{3} 3 k(b+d)}{\cosh ^{3} k d} \sin (k a-\sigma t) .
\end{aligned}
$$

and

$\phi_{3}^{\prime}=D_{3}^{\prime}\left(\sigma_{0} t\right), \quad \alpha \cdot \frac{\omega_{2}}{\sigma_{0}}+\lambda_{3} \cdot \operatorname{sech}^{2} k d=0$.

The wave pressure can thus be given by

$$
\begin{aligned}
& \frac{p_{3}}{\rho}=\left\{\beta _ { 3 } \left[3 \frac{\sigma_{0}^{2}}{k} \frac{\cosh 3 k(b+d)}{\cosh ^{3} k d}\right.\right. \\
& \left.-g \frac{\sinh 3 k(b+d)}{\cosh ^{3} k d}\right]-\frac{1}{2} \alpha\left(7 \beta_{2}-\alpha^{2} k\right) \sigma_{0}^{2} \frac{\cosh k(b+d)}{\cosh ^{3} k d} \\
& \left.+\frac{1}{2} \alpha k g \beta_{2} \frac{\sinh ^{3} k(b+d)}{\cosh ^{3} k d}\right\} \cdot \cos 3(k a-\sigma t) \\
& -\sigma_{0} D_{3 \sigma_{0} t}^{\prime}+\left\{\frac{3}{2} \alpha \beta_{2} \sigma_{0}^{2} \frac{\cosh 3 k(b+d)}{\cosh ^{3} k d}\right. \\
& -\frac{1}{2} \alpha k g\left(3 \beta_{2}+\frac{1}{2} \alpha^{2} k\right) \frac{\sinh ^{3} k(b+d)}{\cosh ^{3} k d} \\
& +\left(\sigma_{0} \frac{\alpha}{k} \omega_{2} \cdot \cosh ^{2} k d-\frac{1}{4} \alpha^{3} k \sigma_{0}^{2}\right) \frac{\cosh k(b+d)_{\cosh ^{3} k d}^{\cosh ^{3} k d}}{\left.-g \lambda_{3} \frac{\sinh k(b+d)}{\cosh ^{3} k d}\right\} \cdot \cos (k a-\sigma t) .}
\end{aligned}
$$

The procedure to obtain the solutions at this order is similar to that of $O\left(\varepsilon^{2}\right)$. The secular terms that grow with time have to be zero. Using zero pressure condition at the free surface $\left(p_{3}=0\right.$ at $\left.b=0\right)$, we obtain

$\alpha \omega_{2}+\sigma_{0} \lambda_{3} \operatorname{sech}^{2} k d=0$,

From Eq. (74), we get the solutions of $\beta_{3}, \lambda_{3}$ and $\omega_{2}$

$\beta_{3}=\frac{1}{64} \alpha^{3} k^{2}\left(9 \tanh ^{-4} k d-22 \tanh ^{-2} k d+13\right)$,

$\lambda_{3}=-\frac{1}{16} \alpha^{3} k^{2}\left(9 \tanh ^{-2} k d-10+9 \tanh ^{2} k d\right) \cosh ^{2} k d$,

$\omega_{2}=\frac{1}{16} \alpha^{2} k^{2}\left(9 \tanh ^{-2} k d-10+9 \tanh ^{2} k d\right) \cdot \sigma_{0}$,

$D_{3}^{\prime}\left(\sigma_{0} t\right)=$ arbitrary constant $=0$.

Finally, the physical parameters to the third-order solutions in Lagrangian form are given as follows:

$$
\begin{aligned}
& f_{3}=\left[-\beta_{3} \frac{\cosh 3 k(b+d)}{\cosh ^{3} k d}+\frac{1}{6} \alpha k\left(5 \beta_{2}-\frac{1}{2} \alpha^{2} k\right) \frac{\cosh k(b+d)}{\cosh ^{3} k d}\right] \sin 3(k a-\sigma t) \\
& -\left[\frac{1}{2} \alpha k\left(5 \beta_{2}+\alpha^{2} k\right) \frac{\cosh 3 k(b+d)}{\cosh ^{3} k d}+\lambda_{3} \frac{\cosh k(b+d)}{\cosh ^{3} k d}\right] \sin (k a-\sigma t), \\
& g_{3}=\left[\beta_{3} \frac{\sinh 3 k(b+d)}{\cosh ^{3} k d}-\frac{1}{2} \alpha k \beta_{2} \frac{\sinh k(b+d)}{\cosh ^{3} k d}\right] \cos 3(k a-\sigma t) \\
& +\left[\frac{1}{2} \alpha k\left(3 \beta_{2}+\frac{1}{2} \alpha^{2} k\right) \frac{\sinh 3 k(b+d)}{\cosh ^{3} k d}\right. \\
& \left.+\lambda_{3} \frac{\sinh k(b+d)}{\cosh ^{3} k d}\right] \cos (k a-\sigma t) \text {, } \\
& f_{3}^{\prime}=g_{3}^{\prime}=\phi_{3}^{\prime}=0 \text {, } \\
& \phi_{3}=U \cdot\left[-\beta_{3} \frac{\cosh 3 k(b+d)}{\cosh ^{3} k d}+\frac{1}{6} \alpha k\left(5 \beta_{2}-\frac{1}{2} \alpha^{2} k\right) \frac{\cosh k(b+d)}{\cosh ^{3} k d}\right] \\
& \cdot \sin 3(k a-\sigma t)-U \cdot\left[\frac{1}{2} \alpha k\left(5 \beta_{2}+\alpha^{2} k\right) \frac{\cosh 3 k(b+d)}{\cosh ^{3} k d}\right. \\
& \left.+k \lambda_{3} \frac{\cosh k(b+d)}{\cosh ^{3} k d}\right] \cdot \sin (k a-\sigma t) \\
& +\frac{\sigma_{0}}{k} \beta_{3} \frac{\cosh 3 k(b+d)}{\cosh ^{3} k d} \sin 3(k a-\sigma t) \\
& -\frac{1}{2} \alpha\left(3 \beta_{2}-\frac{1}{2} \alpha^{2} k\right) \cdot \sigma_{0} \cdot \frac{\cosh k(b+d)}{\cosh ^{3} k d} \sin 3(k a-\sigma t) \\
& +\frac{1}{2} \alpha \beta_{2} \sigma_{0} \frac{\cosh 3 k(b+d)}{\cosh ^{3} k d} \sin (k a-\sigma t) \text {, } \\
& \sigma_{2}=-\frac{1}{2} \alpha^{2} k^{2} \sigma_{0} \frac{\cosh 2 k(b+d)}{\cosh ^{2} k d} \\
& +\frac{1}{16} \alpha^{2} k^{2}\left(9 \tanh ^{-2} k d-10+9 \tanh ^{2} k d\right) \cdot \sigma_{0}, \\
& \frac{p_{3}}{\rho}=\left\{\beta_{3}\left[3 \frac{\sigma_{0}^{2}}{k} \frac{\cosh 3 k(b+d)}{\cosh ^{3} k d}-g \frac{\sinh 3 k(b+d)}{\cosh ^{3} k d}\right]\right. \\
& -\frac{1}{2} \alpha\left(7 \beta_{2}-\alpha^{2} k\right) \sigma_{0}^{2} \frac{\cosh k(b+d)^{2}}{\cosh ^{3} k d} \\
& \left.+\frac{1}{2} \alpha k g \beta_{2} \frac{\sinh k(b+d)}{\cosh ^{3} k d}\right\} \cdot \cos 3(k a-\sigma t) \\
& +\left\{\frac{3}{2} \alpha \beta_{2} \sigma_{0}^{2} \frac{\cosh 3 k(b+d)}{\cosh ^{3} k d}\right. \\
& -\frac{1}{2} \alpha k g\left(3 \beta_{2}+\frac{1}{2} \alpha^{2} k\right) \frac{\sinh 3 k(b+d)}{\cosh ^{3} k d} \\
& +\left(\sigma_{0} \frac{\alpha}{k} \omega_{2} \cdot \cosh ^{2} k d\right. \\
& \left.-\frac{1}{4} \alpha^{3} k \sigma_{0}^{2}\right) \frac{\cosh k(b+d)}{\cosh ^{3} k d} \\
& \left.-g \lambda_{3} \frac{\sinh k(b+d)}{\cosh ^{3} k d}\right\} \cdot \cos (k a-\sigma t) \text {. }
\end{aligned}
$$

Equation (80) is the second-order angular frequency correction for a particle, in which the first term is the second-order Stokes wave frequency and the second term varies monotonically with the vertical label $b$ or the wavelength-averaged level of the particles. The result differs from the Eulerian wave frequency. The third-order solutions of Eqs. (76) and (77) are periodic functions and have a combination of both 
first and third harmonic components. Thus, the solution of system has the following expressions:

$x=a+U t+\varepsilon f_{1}(a, b, \sigma t)+\varepsilon^{2}\left[f_{2}(a, b, \sigma t)\right.$

$\left.+f_{2}^{\prime}\left(a, b, \sigma_{0} t\right)\right]+\varepsilon^{3} f_{3}(a, b, \sigma t)$,

$y=b+\varepsilon g_{1}(a, b, \sigma t)+\varepsilon^{2} g_{2}(a, b, \sigma t)+\varepsilon^{3} g_{3}(a, b, \sigma t)$,

$\phi=U a+\frac{1}{2} U^{2} t+\varepsilon \phi_{1}(a, b, \sigma t)$

$+\varepsilon^{2}\left[\phi_{2}(a, b, \sigma t)+\phi_{2}^{\prime}\left(a, b, \sigma_{0} t\right)\right]+\varepsilon^{3} \phi_{3}(a, b, \sigma t)$,

$p=-\rho g b+\varepsilon p_{1}(a, b, \sigma t)+\varepsilon^{2} p_{2}(a, b, \sigma t)+\varepsilon^{3} p_{3}(a, b, \sigma t)$,

$\sigma=\sigma_{0}(a, b)+\varepsilon^{2} \sigma_{2}(a, b)=2 \pi / T_{\mathrm{L}}$

The set of Eqs. (82)-(86) ensures that Bernoulli's condition of constant pressure is satisfied on the free surface.

\section{Experimental setup and results}

The aim of this experiment is to quantitatively investigate the characteristics of the water particle for periodic progressive gravity waves in uniform water depth. The experiments of particle trajectory beneath wave-current interaction have been carried out at the hydraulic laboratory of the Department of Marine Environment and Engineering of National Sun Yat-Sen University. The experimental setup, which comprises two interacting systems (1) a flume for the wave generation and propagation and (2) a recirculating apparatus, allows for a current to be generated. The produced current is nearly uniform with a variation of $\pm 6.7 \%$. The wave flume is $35 \mathrm{~m}$ long, $1 \mathrm{~m}$ wide and $1.2 \mathrm{~m}$ high, with a fixed horizontal bottom. The waves are generated by means of a piston wavemaker, which is driven by a pneumatic system and is electronically controlled. The surface elevation is measured by means of several resistance wave gauges. An electromagnetic current meter (ACM-200A) is used to measure the current velocity. A camera was set up in front of the glass wall about $9.0 \mathrm{~m}$ from the wave generator to capture the particle motion. Four wave gauges were located at $7.0 \mathrm{~m}, 15 \mathrm{~m}$, $16 \mathrm{~m}$ and $16.6 \mathrm{~m}$ from the wave generator to measure the incident waves. At the end of the tank, a 1:10 slope rubberizedfiber wave-absorbing beach was built to prevent the reflected wave.

The orbital experiments (polysterne (PS) particle with diameter about $1 \mathrm{~mm}$ and density near $1.05 \mathrm{~g} \mathrm{~cm}^{-3}$ ) were conducted at two constant water depths $d(50 \mathrm{~cm}$ and $80 \mathrm{~cm})$ and the various wave periods $T_{\mathrm{E}}(0.96-2.06 \mathrm{~s})$. The wave height $H$, which was the mean crest-to trough wave height computed over 20 different waves after the generated progressive waves became stable (about 7 waves), was varied over a range of about $3.17-15.2 \mathrm{~cm}$. The particle motion was measured at different positions from the still water level to about $10.5 \mathrm{~cm}$ depth. In Table 1 , the values of the control parameters, namely the current velocity $\mathrm{U}$, the wave height $\mathrm{H}$, and wave period $T_{\mathrm{E}}$ are reported along with other Lagrangian quantities parameters which were the mean value of three different measurements (the particle motion period $T_{\mathrm{L}}$, mass transport velocity and Lagrangian mean level). It shows an excellent agreement for the Lagrangian properties of water particle (Lagrangian wave frequency, mass transport and Lagrangian mean level) between the present third-order solution and experimental data.

\section{Results and discussions}

\subsection{Mass transport velocity}

Taking time-average over one Lagrangian wave period to the terms of the horizontal particle displacement, the so-called drift velocity, over the whole range of depths can be obtained as follows:

$$
\begin{aligned}
& \frac{\bar{x}_{t}}{c_{0}}=\frac{U+\overline{\sum_{n=1}^{3} \varepsilon^{n}\left[f_{n t}(a, b, \sigma t)+f_{n t}^{\prime}\left(a, b, \sigma_{0} t\right)\right]}}{c_{0}} \\
& =U+\frac{1}{2} \alpha^{2} k^{2}\left(1+\tanh ^{2} k d\right) \frac{\cosh 2 k(b+d)}{\cosh 2 k d}, \quad c_{0}=\frac{\sigma_{0}}{k},
\end{aligned}
$$

where the overbar denotes time-average over a Lagrangian wave period, i.e., the period of particle motion, where $c_{0}$ is the linear phase speed. The second term of Eq. (87) on the right-hand side, a second-order correction quantity, is the same as that obtained by Longuet-Higgins (1953) as $U=0$. From Eq. (87), the mass transport velocity is a function of the wave steepness, the water depth, uniform current and the vertical Lagrangian label. Differentiating Eq. (87) with respect to the vertical Lagrangian label $b$ shows that the secondorder drift velocity is always positive but monotonically decays with depth from the surface to the bottom. In Fig. 2, the mass transport velocity is plotted against the water depth and the uniform current in the wave-current interaction field. From Fig. 2, we can clearly see that uniform current has a significant effect on the drift velocity. It can be seen that the effect of increasing current velocity is generally to augment the magnitude and extent of the time-averaged drift velocity, thus resulting in large horizontal distance traveled by a particle compared with the case without uniform current. The increasing of following current is to enhance the magnitude of the drift velocity over the whole range of depths, and a significant amount of fluid that has been transported forward, on the contrary, the drift velocity decreases by the adverse current. It is also remarkable that under the same wave, subsurface particles travel slower and diminish rapidly with the vertical position below the free surface. 
Table 1. Experimental conditions and comparison of measured and theoretical results of the particle period $T_{\mathrm{L}}$, mass transport velocity $U_{M}=\bar{x}_{t}-U$ and Lagrangian mean wave level $\bar{\eta}_{L}$.

\begin{tabular}{|c|c|c|c|c|c|c|c|c|c|c|c|c|c|}
\hline \multirow[t]{2}{*}{ No } & \multirow[t]{2}{*}{$T_{\mathrm{E}}(\mathrm{s})$} & \multirow[t]{2}{*}{$d(\mathrm{~cm})$} & \multirow[t]{2}{*}{$H(\mathrm{~cm})$} & \multirow[t]{2}{*}{$b(\mathrm{~cm})$} & \multirow[t]{2}{*}{$U(\mathrm{~cm})$} & \multirow[t]{2}{*}{$H / L$} & \multirow[t]{2}{*}{$d / L$} & \multicolumn{2}{|c|}{$T_{\mathrm{L}}(s)$} & \multicolumn{2}{|c|}{$U_{M}(b)\left(\mathrm{cms}^{-1}\right)$} & \multicolumn{2}{|c|}{$\bar{\eta}_{L}(\mathrm{~cm})$} \\
\hline & & & & & & & & Measured & Theory & Measured & Theory & Measured & Theory \\
\hline a & 0.99 & 50 & 4.62 & 0 & 2.95 & 0.031 & 0.333 & 1.002 & 1.000 & 4.41 & 4.38 & 0.12 & 0.11 \\
\hline $\mathrm{b}$ & 1.00 & 80 & 13.20 & 0 & 9.63 & 0.080 & 0.485 & 1.061 & 1.063 & 19.12 & 19.15 & 0.77 & 0.78 \\
\hline $\mathrm{c}$ & 1.39 & 80 & 15.20 & 0 & 8.93 & 0.052 & 0.274 & 1.433 & 1.430 & 14.63 & 14.59 & 0.65 & 0.63 \\
\hline $\mathrm{d}$ & 2.06 & 80 & 5.82 & 0 & 11.72 & 0.012 & 0.158 & 2.062 & 2.064 & 12.12 & 12.15 & 0.06 & 0.07 \\
\hline e & 0.96 & 50 & 5.23 & 0 & -5.94 & 0.037 & 0.349 & 0.979 & 0.976 & -4.03 & -3.98 & 0.17 & 0.15 \\
\hline$f$ & 0.96 & 50 & 6.81 & 0 & -6.60 & 0.048 & 0.349 & 0.982 & 0.979 & -3.36 & -3.31 & 0.26 & 0.25 \\
\hline g & 1.66 & 50 & 4.20 & -9.5 & -7.14 & 0.013 & 0.155 & 1.661 & 1.663 & -6.79 & -6.83 & 0.03 & 0.04 \\
\hline $\mathrm{h}$ & 0.93 & 50 & 3.17 & 0 & -21.01 & 0.024 & 0.376 & 0.936 & 0.934 & -20.25 & -20.21 & 0.08 & 0.06 \\
\hline
\end{tabular}

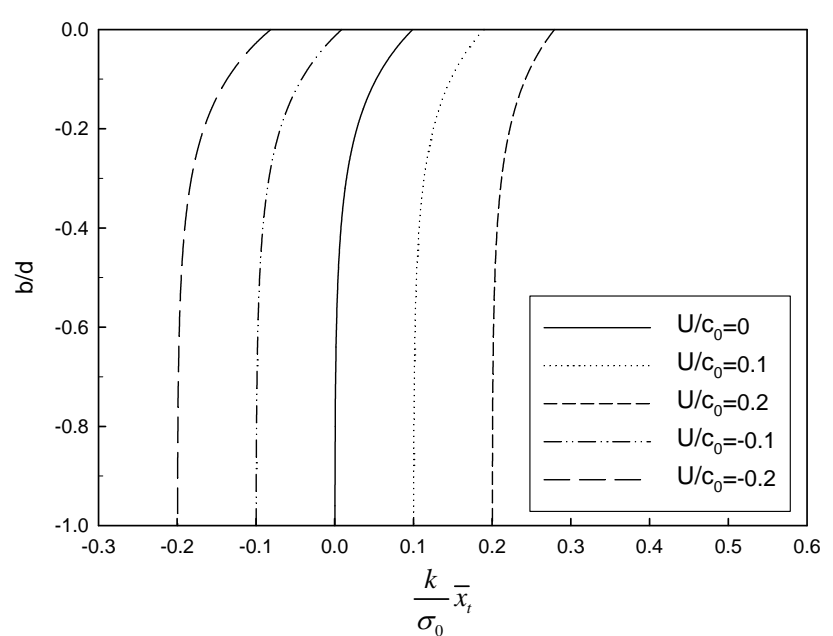

Fig. 2. The dimensionless mass transport velocity profile $\frac{\bar{x}_{t}}{c_{0}}$ at the relative water depth $d / L=0.5$ and the relative wave height $H / L=$ 0.1 under various current conditions.

\subsection{Lagrangian wave frequency}

The Lagrangian angular frequency $\sigma$ up to third order can also be obtained as

$$
\begin{aligned}
& \sigma=\sigma_{0}+\sigma_{2}(b) \\
& =\sigma_{0}-\frac{1}{2} \alpha^{2} k^{2}\left(1+\tanh ^{2} k d\right) \frac{\cosh 2 k(b+d)}{\cosh 2 k d} \sigma_{o} \\
& -\sigma_{0} \frac{\lambda_{3}}{\alpha} \operatorname{sech}^{2} k d .
\end{aligned}
$$

Hence, a general Lagrangian wave period $\sigma$ differing from the Eulerian wave period for all particles at different vertical level $b$ can be obtained directly in the odd-order Lagrangian solutions. The difference between the Lagrangian frequency $\sigma$ and the Eulerian wave frequency $\sigma_{E}$ is

$$
\begin{aligned}
& \sigma-\sigma_{E}=-\frac{1}{2} \alpha^{2} k^{2}\left(1+\tanh ^{2} k d\right) \frac{\cosh 2 k(b+d)}{\cosh 2 k d} \sigma_{o}, \\
& \sigma_{E}=\sigma_{0}-\sigma_{0} \frac{\lambda_{3}}{\alpha} \operatorname{sech}^{2} k d,
\end{aligned}
$$

where $\sigma_{E}$ is the angular frequency computed by Stokes expansion in the Eulerian system. It can be shown that Eq. (89)

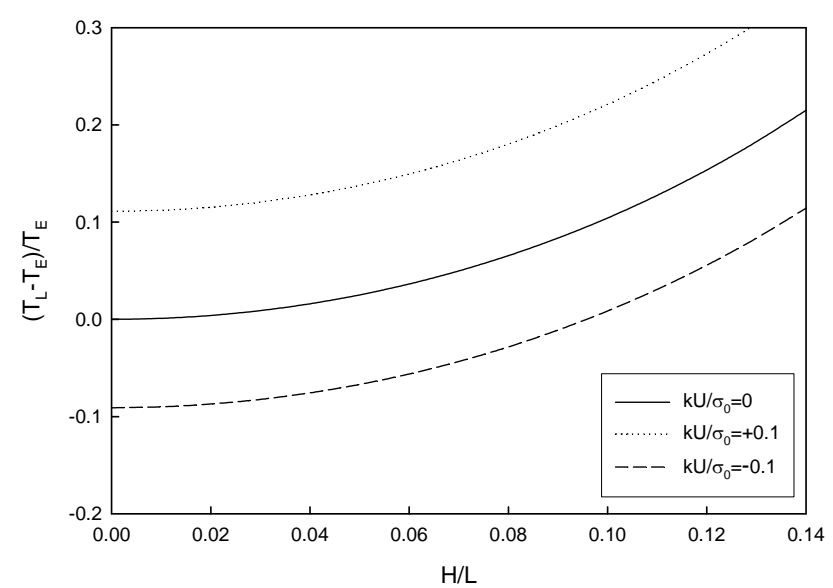

Fig. 3. The relative ratio for water particle motion at the free surface between Lagrangian and Eulerian periods for three current conditions.

calculates the resultant wave period in Lagrangian form in a combined flow field for all the water particles at different elevations within the fluid domain. This equation also indicates the frequency of particle motion near the surface is smaller than that at the subsurface. For water particle motion at the free surface, the relative ratio between the Lagrangian form $T_{\mathrm{L}}=2 \pi / \sigma_{L}$ and Eulerian form $T_{\mathrm{E}}=2 \pi / \sigma_{E}$ for three different current conditions is shown in Fig. 3 , in which $T_{\mathrm{L}} / T_{\mathrm{E}}$ is found to increase with a following current (positive $U$ ), and to decrease in an opposing current (negative $U$ ) for a given wave steepness $H / L$. This implies that for a coplanar flow the water particles near the surface move forward further over one wave cycle than those against an opposing flow. Moreover, $T_{\mathrm{L}}$ is larger than $T_{\mathrm{E}}$, even with the wave alone (e.g., the case of $U=0$ in Fig. 3). 


\subsection{Lagrangian mean level}

Averaging the particle elevation up to the third order over a given period of particle motion, the present theory gives the Lagrangian mean level $\bar{\eta}_{L}(b)$, which is higher than the Eulerian mean level $\bar{\eta}_{E}=0$ as

$\bar{\eta}_{L}-\bar{\eta}_{E}=\frac{1}{T_{\mathrm{L}}} \int_{0}^{T_{\mathrm{L}}} y \mathrm{~d} t=\frac{1}{4} \alpha^{2} k \frac{\sinh 2 k(b+d)}{\cosh ^{2} k d}$.

Longuet-Higgins $(1979,1986)$ also showed that the Lagrangian mean level is higher than the Eulerian mean level for progressive water waves. However, his expression is applicable only to particles at the free surface and is the same as that given by the first term of Eq. (90) at $b=0$.

\subsection{Wave profiles and water particle orbits}

The most important characteristic of fluid motion described by the Lagrangian solution is the trajectories of particles which are represented by Eqs. (82) and (83). The parameter $\alpha$ can be determined by the wave height $H$ defined as half the vertical distance between the wave crest and wave trough, in wave number $k$ and the water depth $d$ given. Hence, we have

$$
\frac{H}{2}=\left[g_{1}+g_{3}\right]_{b=0, k a-\sigma t=2 n \pi}, \quad n \in I
$$

the horizontal and vertical particle trajectories are

$x=a+U t+\sum_{n=1}^{3}\left(f_{n}+f_{n}^{\prime}\right), \quad y=b+\sum_{n=1}^{3}\left(g_{n}+g_{n}^{\prime}\right)$.

Figure 4 provides a comparison of the wave profiles between Lagrangian and Eulerian solutions, both to a thirdorder approximation. The results reveal that the height of the wave increases against an opposing current (negative $F_{\mathrm{r}}=U / c_{0}$ ) and decreases on a following current (positive $F_{\mathrm{r}}$ ). In Figure $4 \mathrm{a}$, the Eulerian wave profiles have anomalous bumps in the trough for the wave conditions tested, which may not be a realistic physical phenomenon for waves of constant form. On the other hand, the Lagrangian wave profiles have sharper crests and broader troughs, as well as exclude any artificial bumps at or near the trough. Clearly the thirdorder Lagrangian solution is more exact than the Eulerian solution of the same order for describing the shape of the gravity wave. In general, the surface profile is an unknown function in the Eulerian approach, and the boundary conditions at the free surface can only be satisfied in an approximately manner. However, the free surface in the Lagrangian description is represented explicitly by a parametric function for the particles. The advantage of using Lagrangian description is that it allows flexibility for capturing the actual shape and the wave kinematics above mean water level. Thus theoretically, Lagrangian solution can provide better prediction for the wave profile at a large Froude number than the Stokes' expansion to the same order. The wave profiles depicted in (a)

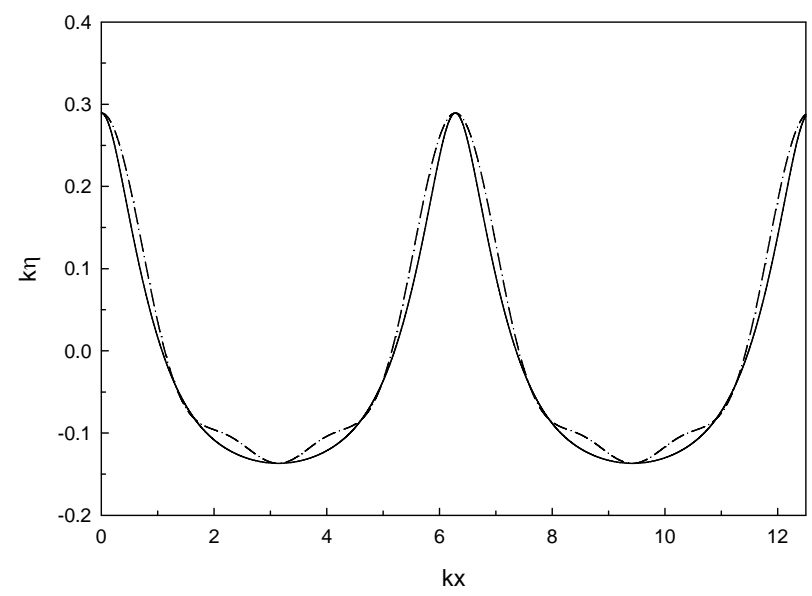

(b)

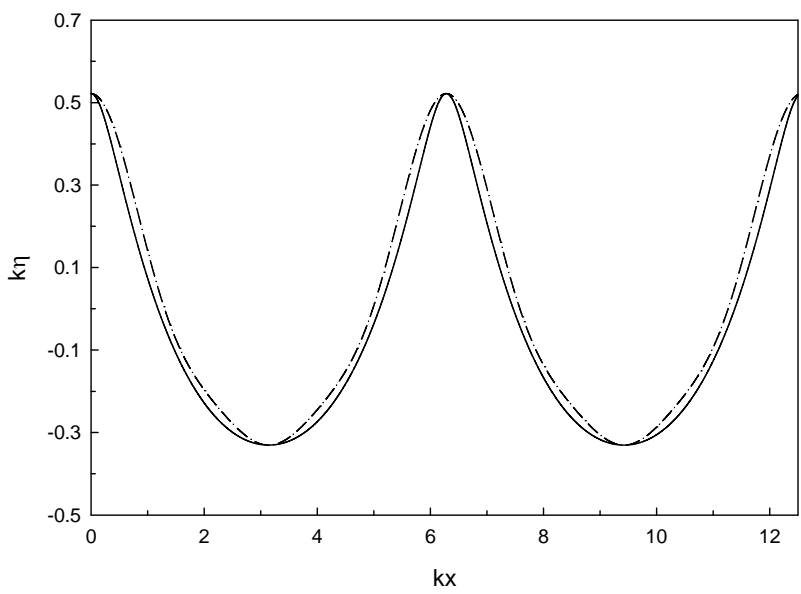

Fig. 4. A comparison on the wave profiles for the Eulerian and Lagrangian solutions both to a third-order under different current conditions (a) $F_{\mathrm{r}}=0.3$, (b) $F_{r}=-0.2$. Wave conditions $d / L=0.2$ and $H / L=0.1$ (Solid line: third-order Lagrangian solution; dashdotted line: third-order Eulerian solution).

Fig. 4 also show that they are symmetric with respect to the crest line, which were recently proven to hold true for irrotational waves

In Fig. 5a-h, water particle orbits plotted for different current magnitudes exhibit variations in orbital patterns, both in shapes and sizes, as a function of its original elevation. As can be expected in Fig. 5, the orbital displacement based on a third order solution is non-closed for a pure progressive wave. The elongation or shortening of the orbits in the case with following or opposing current is apparent in Fig. 5a-h. Their orbital dimensions in the cases with positive $U$ values reflect the magnitude of a following current, and the converse is true for the condition with opposing current. It can be seen in each of the orbits plotted that a water 
(a) $T_{E}=0.99 \mathrm{sec}, \mathrm{H}=4.62 \mathrm{~cm}, \mathrm{~d}=50 \mathrm{~cm}, \mathrm{~b}=0 \mathrm{~cm}, \mathrm{U}=2.95 \mathrm{~cm} / \mathrm{sec}$

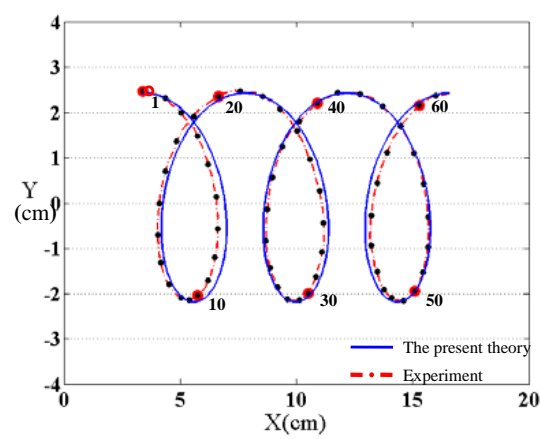

(c) $T_{E}=1.39 \mathrm{sec}, \mathrm{H}=15.20 \mathrm{~cm}, \mathrm{~d}=80 \mathrm{~cm}, \mathrm{~b}=0 \mathrm{~cm}, \mathrm{U}=8.93 \mathrm{~cm} / \mathrm{sec}$

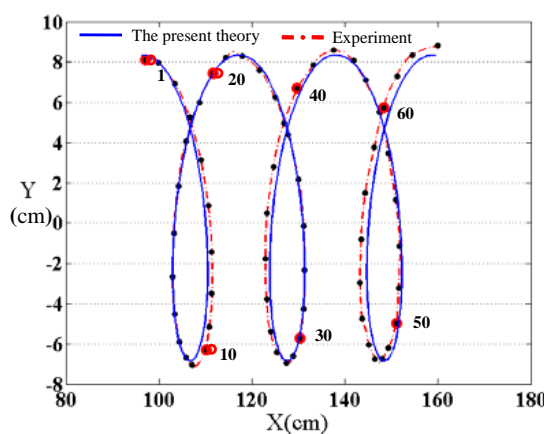

(e) $T_{E}=0.96 \mathrm{sec}, \mathrm{H}=5.23 \mathrm{~cm}, \mathrm{~d}=50 \mathrm{~cm}, \mathrm{~b}=0 \mathrm{~cm}, \mathrm{U}=-5.94 \mathrm{~cm} / \mathrm{sec}$

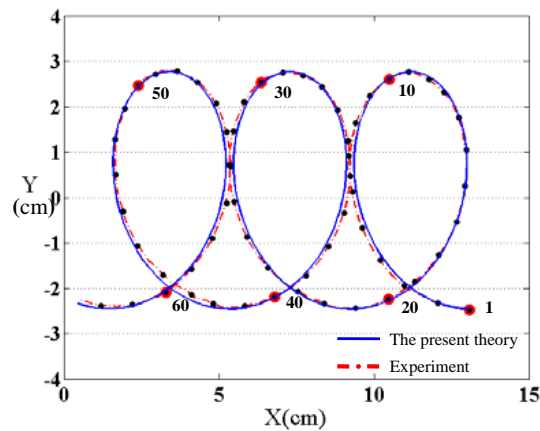

(g) $T_{E}=1.66 \mathrm{sec}, \mathrm{H}=4.20 \mathrm{~cm}, \mathrm{~d}=50 \mathrm{~cm}, \mathrm{~b}=-9.5 \mathrm{~cm}, \mathrm{U}=-7.14 \mathrm{~cm} / \mathrm{sec}$

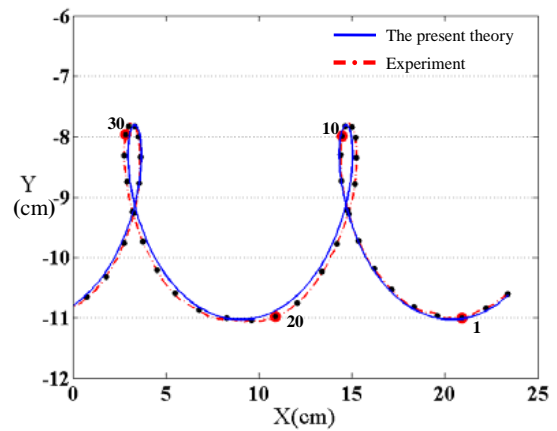

(b) $T_{\mathrm{E}}=1 \mathrm{sec}, \mathrm{H}=13.20 \mathrm{~cm}, \mathrm{~d}=80 \mathrm{~cm}, \mathrm{~b}=0 \mathrm{~cm}, \mathrm{U}=9.63 \mathrm{~cm} / \mathrm{sec}$

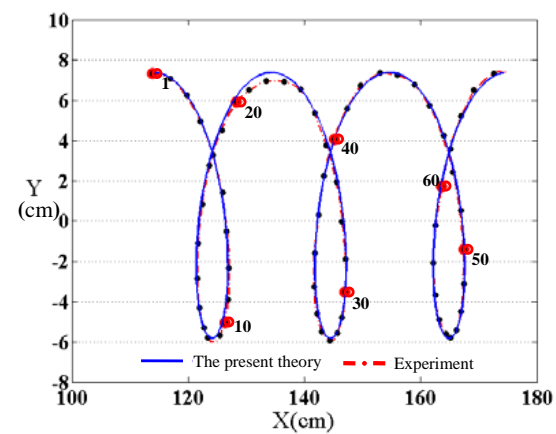

(d) $T_{E}=2.06 \mathrm{sec}, \mathrm{H}=5.82 \mathrm{~cm}, \mathrm{~d}=80 \mathrm{~cm}, \mathrm{~b}=0 \mathrm{~cm}, \mathrm{U}=11.72 \mathrm{~cm} / \mathrm{sec}$

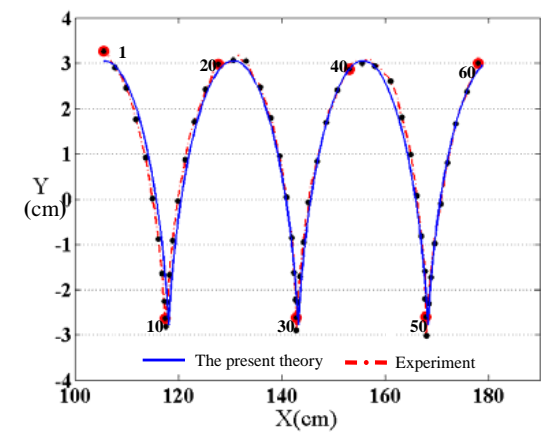

(f) $T_{E}=0.96 \mathrm{sec}, \mathrm{H}=6.81 \mathrm{~cm}, \mathrm{~d}=50 \mathrm{~cm}, \mathrm{~b}=0 \mathrm{~cm}, \mathrm{U}=-6.60 \mathrm{~cm} / \mathrm{sec}$

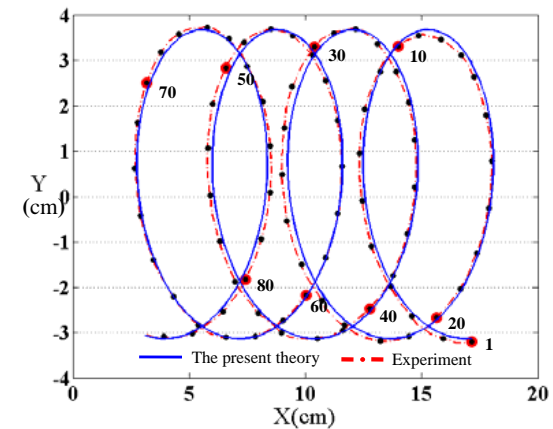

(h) $T_{E}=0.93 \mathrm{sec}, \mathrm{H}=3.17 \mathrm{~cm}, \mathrm{~d}=50 \mathrm{~cm}, \mathrm{~b}=0 \mathrm{~cm}, \mathrm{U}=-21.01 \mathrm{~cm} / \mathrm{sec}$

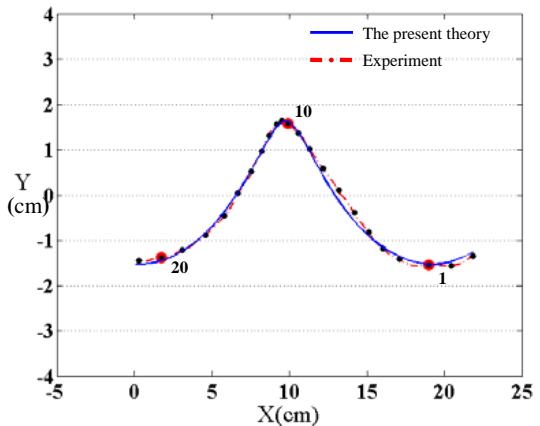

Fig. 5. Comparisons between the orbits of water particles obtained by the presented theory and those from the experimental measurements of the PS motions at different water levels $b$ in the various experimental wave cases, where solid line is the theoretical result and point is the experimental data which the time interval between two adjacent points is $T_{\mathrm{E}} / 20, T_{\mathrm{E}}$ is the wave period. 
particle advances a distance forward, which is commonly referred to as mean horizontal drift or mass transport in the direction of wave propagation. The water particle at the free surface $(b=0)$ travels fastest, whilst that in the interior of the fluid propagates slower. To the third-order approximation, the particle trajectory has non-closed orbit, irrespective of their initial mean locations. This confirms the theoretical results obtained in Constantin (2006) and Constantin and Strauss (2010) for waves of large amplitude.

In the case of wave on a following current, the effect of increasing current speed is generally to augment the magnitude and extent of the time-averaged drift velocity, thus resulting in large horizontal distance traveled by a particle compared with the case without current. Again, the converse is true when a wave train encounters an opposing current, which retards the advancement of water particles compared to that without a current or with a following current. As the strength of an opposing current becomes comparable with the wave speed, the water particle at greater depths beneath the still water level is mainly transport by the opposing current in larger current velocity and the direction of particle movement becomes contrary to wave progression. Figure $5 \mathrm{a}-\mathrm{h}$ show excellent agreements between the measured trajectory and the theoretical trajectory predicted by the present third order Lagrangian wave theory. This is also in agreement with the theoretical findings in Constantin and Strauss (2010).

\section{Conclusions}

A particle-specific description of irrotational finite-amplitude progressive gravity waves on a uniform current in water of uniform depth satisfying all the governing equations and the boundary conditions is presented. The new Lagrangian solution is obtained to the third order. It can be used not only to determine the wave properties available in the Eulerian solution, but also to get the trajectory, the period, the mass transport and the Lagrangian mean level of a water particle, which are not available from the Eulerian solution. In the Lagrangian solution to a second-order, the Lagrangian mean level of a particle orbit over its motion period is found to be higher than that of the Eulerian, and it also has a timedependent term referred to as the mass transport velocity, which is applicable to the entire flow field. The frequency associated with water particle motions in Lagrangian form differs from that of the Eulerian, and the former is a function of wave steepness, uniform current speed and the Lagrangian vertical marked label $b$ for each individual particle that can be obtained directly based on the third-order solutions.

From the trajectories of water particles resulting from wave-current interaction, it is found that particle displacement near the surface decreases due to its mass transport velocity is resisted by an opposing current. Again in the case with an opposing current, the water particle further beneath the still water level is mainly transported by the op- posing current in the direction against the progressive wave, especially with a current in the large Froude number $F_{r}$. In the cases with a following current, the effect of increasing current speed is generally to increase the magnitude of the time-averaged mass transport velocity since the current is in the same direction as the wave propagation, thus resulting in augmentation to the horizontal distance traveled by a particle. Finally, a set of experiments analyzing the Lagrangian properties of nonlinear wave-current interaction flow is conducted in the wave tank. It shows excellent agreement between the experimental and theoretical results, including particle trajectories, Lagrangian wave frequency, mass transport velocity and Lagrangian mean water level predicted by the present third-order Lagrangian solution.

Acknowledgements. The authors thank the anonymous referees for invaluable comments. This work was supported in part by the NSC grants 101-2911-I-110-504, 101-2622-E-006-010-CC2 and 99-2923-E-110-001-MY3 in Taiwan.

Edited by: C. Kharif

Reviewed by: H. B. Branger and another anonymous referee

\section{References}

Biesel, F.: Study of wave propagation in water of gradually varying depth, Gravity Waves, US National Bureau of Standards, Circular 521, 243-253, 1952.

Brevik, I.: Flume experiment on waves and current II, Smooth bed, Coast. Eng., 4, 149-177, 1980.

Buldakov, E. V., Taylor, P. H., and Eatock Taylor, R.: New asymptotic description of nonlinear water waves in Lagrangian coordinates, J. Fluid Mech., 562, 431-444, 2006.

Chen, Y. Y., Hsu, H. C., Chen, G. Y., and Hwung, H. H.: Theoretical analysis of surface waves shoaling and breaking on a sloping bottom, Part 2 Nonlinear waves, Wave Motion, 43, 356-369, 2006.

Chen, Y. Y. and Hsu, H. C.: Third-order asymptotic solution of nonlinear standing water waves in Lagrangian coordinates, Chinese Physics B., 18, 861, 2009.

Chen, Y. Y., Hsu, H. C., and Chen, G. Y.: Lagrangian experiment and solution for irrotational finite-amplitude progressive gravity waves at uniform depth, Fluid Dyn. Res., 42, 045511, doi:10.1088/0169-5983/42/4/045511, 2010.

Clamond, D.: On the Lagrangian description of steady surface gravity waves, J. Fluid Mech. 589, 433-454, 2007.

Constantin, A.: Edge waves along a sloping beach, J. Phys. A, 34, 9723-9731, 2001.

Constantin, A.: On the deep water wave motion, J. Phys. A, 34, 1405-1417, 2001.

Constantin, A. and Strauss, W.: Exact steady periodic water waves with vorticity, Commun. Pure Appl. Math., 57, 481-527, 2004.

Constantin, A.: The trajectories of particles in Stokes waves, Invent. Math. 166, 523-535, 2006.

Constantin, A.: On the particle paths in solitary water waves, Q. Appl. Math., 68, 81-90,2010.

Constantin, A. and Strauss, W.: Pressure beneath a Stokes wave, Commun Pure Appl. Math., 63, 533-557, 2010. 
Constantin, A. and Escher, J.: Analyticity of periodic traveling free surface water waves with vorticity, Ann. Math., 173, 559-568, 2011.

Gerstner, F. J.: Theorie de wellen, Abh. d. K. bohm. Ges. Wiss., reprinted in Ann der Physik 1809, 32, 412-440, 1802.

Hsu, H. C., Chen, Y. Y., and Wang, C. F.: Perturbation analysis of short-crested waves in Lagrangian coordinates, Nonlinear Analysis: Real World Application, 11, 1522-1536, 2010.

Jonsson, I. G.: Wave-current interaction, The Sea, Ocean Eng. Series, 9, 1990.

Kemp, P. H. and Simons R. R.: The interactions of waves and a turbulence current: waves propagating with the current, J. Fluid Mech., 116, 227-250, 1982.

Kemp, P. H. and Simons R. R.: The interactions of waves and a turbulence current: waves propagating against the current, J. Fluid Mech., 130, 73-89, 1988.

Lamb, H.: Hydrodynamics, 6th ed. Cambridge University Press, 1932.

Longuet-Higgins, M. S.: Mass transport in water waves, Philos. T. R. Soc. A, 245, 533-581, 1953.

Longuet-Higgins, M. S.: The trajectories of particles in steep, symmetric gravity waves, J. Fluid Mech., 94, 497-517, 1979.

Longuet-Higgins, M. S.: Eulerian and lagrangian aspects of surface waves, J. Fluid Mech., 173, 683-707, 1986.

Miche, A.: Mouvements ondulatoires de la mer en profondeur constante ou décroissante, Annales des ponts et chaussees, 25-78, 131-164, 270-292, 369-406, 1944.

Naciri, M. and Mei, C. C.: Evolution of a short surface wave on a very long surface wave of finite amplitude, J. Fluid Mech., 235, 415-452, 1992.
$\mathrm{Ng}$, C. O.: Mass transport in gravity waves revisited, J. Geophys., Res., 109, C04012, doi:10.1029/2003JC002121, 2004.

Peregrine, D. H.: Interaction of water waves and currents, Adv. Appl. Mech., 16, 9-117, 1976.

Pierson, W. J.: Perturbation analysis of the Navier-Stokes equations in Lagrangian form with selected linear solution, J. Geophys., Res., 67, 3151-3160, 1962.

Stuhlmeier, R.: On edge waves in stratified water along a sloping beach, J. Nonl. Math. Phys., 18, 127-137, 2011.

Rankine, W. J. M.: On the exact form of waves near the surface of deep water, Philos. T. R. Soc. A, 153, 127-138, 1863.

Sanderson, B.: A Lagrangian solution for internal waves, J. Fluid Mech., 152, 191-137, 1985.

Thomas, G. P.: Wave-current interactions: an experimental and numerical study, Part 1. Linear waves, J. Fluid Mech., 110, 457474, 1981.

Thomas, G. P.: Wave-current interactions: an experimental and numerical study. Part 2, Nonlinear waves, J. Fluid Mech., 216, 505536, 1990.

Thomas, G. P. and Klopman G.: Wave-current interactions in the near-shore region, Adv. Fluid. Mech Ser., Computational Mechanics Publications, 66-84, 1997.

Umeyama, M.: Coupled PIV and PTV measurements of particle velocities and trajectories for surface waves following a steady current, J. Waterw. Port C-ASCE, 137, 85-94, 2010.

Zaman, M. H. and Baddour, E.: Interaction of waves with noncolinear currents, Ocean Eng., 38, 541-549, 2010. 